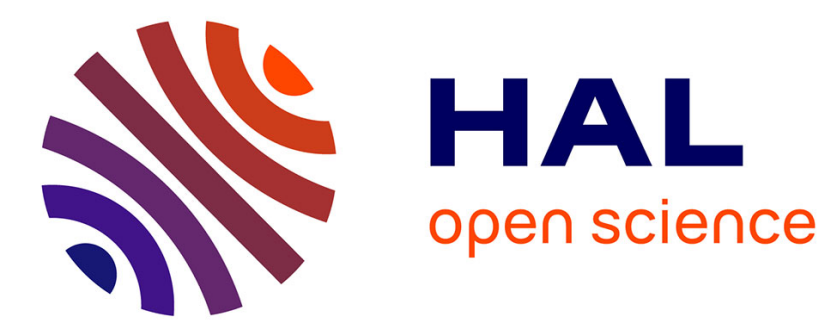

\title{
Et le roi tua l'hippopotame. Enquête sur les origines d'un rite égyptien
}

Aurélie Roche

\section{To cite this version:}

Aurélie Roche. Et le roi tua l'hippopotame. Enquête sur les origines d'un rite égyptien. Archimède: archéologie et histoire ancienne, 2014, 1, pp.71-87. halshs-01586095

\section{HAL Id: halshs-01586095 https://shs.hal.science/halshs-01586095}

Submitted on 12 Sep 2017

HAL is a multi-disciplinary open access archive for the deposit and dissemination of scientific research documents, whether they are published or not. The documents may come from teaching and research institutions in France or abroad, or from public or private research centers.
L'archive ouverte pluridisciplinaire HAL, est destinée au dépôt et à la diffusion de documents scientifiques de niveau recherche, publiés ou non, émanant des établissements d'enseignement et de recherche français ou étrangers, des laboratoires publics ou privés. 


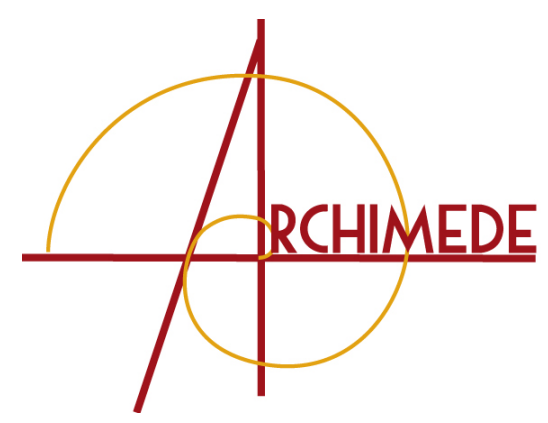

\title{
ET LE ROI TUA L'HIPPOPOTAME. ENQUÊTE SUR LES ORIGINES D'UN RITE ÉGYPTIEN.
}

\author{
Aurélie Roche \\ Doctorante, \\ Université de Strasbourg, \\ Archimède (UMR 7044), \\ aurelie.roche1@gmail.com
}

\section{RÉSUMÉ}

L'enquête débute avec une entrée difficilement déchiffrable de la pierre de Palerme, passage daté du règne de Den, cinquième roi de la première dynastie. La lecture «transpercer l'hippopotame » proposée jusqu'à présent a pu être confortée grâce à l'examen de plusieurs parallèles graphiques conjugué à la présentation de sources contemporaines au règne de Den où une activité similaire est consignée.

L'examen du matériel iconographique et des restes fauniques de l'époque prédynastique révèle que la mise à mort rituelle de l'hippopotame par le roi à l'époque thinite est en réalité I'héritière d'une tradition amorcée dès le Prédynastique par l'élite naissante. Devenue privilège royal, cette pratique rituelle est ensuite perpétuée par la figure royale durant toute la période pharaonique. Sa longévité dénote son rôle fondamental dans le processus de légitimation du pouvoir de Pharaon. Dans ce rituel, I'hippopotame incarne les forces chaotiques de la nature à combattre. En tuant I'animal, le roi harponneur s'approprie son pouvoir et rétablit

\section{Mots-CLÉs}

Hippopotame,

Chasse,

Harpon,

Roi,

Den,

Période thinite,

Pierre de Palerme,

Rite,

Fête,

Mythe d'Horus et Seth. I'ordre sur le chaos. L'importance de ce combat est telle qu'il est rejoué à l'échelle divine dans un mythe fondateur, lorsqu'Horus affronte I'hippopotame Seth pour remporter sa place sur le trône et gouverner l'Égypte.

Mes remerciements s'adressent à Vera Müller pour nos discussions concernant les scellés d'Abydos ainsi qu'à Christine Hue-Arcé pour sa relecture.

The investigation starts with a hardly readable entry from the Palermo Stone, dated back to Den, fifth king of the First Dynasty. This entry has been deciphered as «spearing the hippopotamus » before, and this reading is corroborated here thanks to graphic comparisons and observation of similar activities depicted in items dating from Den's reign.

The study of predynastic iconography and archaeozoological evidence reveals that ritual killing of the hippopotamus by the king during Early Dynastic Egypt results from a predynastic tradition, initiated by the dawning elite. Later to become a privilege of the king, this ritual practice is performed by the royal figure during dynastic times. Its longevity reveals its crucial role in the process leading to legitimate Pharaoh's power. The hippopotamus embodies the chaotic powers that have to be fought. By killing the hippopotamus, the king seizes its destructive power and re-establishes the order over the chaos. This fight is so important that it is played also by gods in a founding myth, when Horus faces Seth the hippo to rule over Egypt.

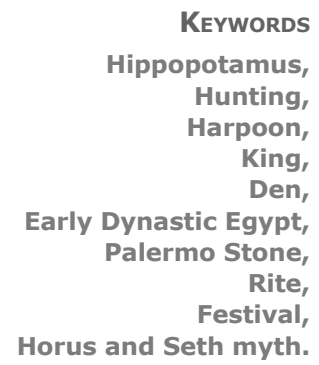


«Taureau des Marais » [2], « cheval de fleuve »[3], « bœuf marin » [4] ou « cheval marin » [5] : voilà autant de qualificatifs qui collent à la peau de I'hippopotame depuis I'Antiquité. Pour comprendre pourquoi le roi se serait risqué à chasser une telle créature, on ne peut cependant réduire son portrait à ces seules épithètes. L'Hippopotamus amphibius est un herbivore vivant en bande, dont l'activité diurne consiste à dormir, tandis qu'il occupe ses nuits à se nourrir dans les herbes sauvages et les champs cultivés le long du Nil [6]. Pachyderme à l'appétit vorace, il peuple le Fayoum et les rivages tout le long de la vallée du Nil et du Delta dès le Paléolithique supérieur [7]. Sa répartition régresse peu à peu jusqu'à sa présence au xix ${ }^{\mathrm{e}}$ siècle en deux seules lentilles, l'une dans le nord du Delta, I'autre au sud d'Assouan [8]. Mais durant l'époque pharaonique, I'animal est encore bien présent et constitue « un terrible fléau par sa manière de se nourrir aux dépens des travailleurs de la terre et des ressources alimentaires du royaume » [9]. Malgré cela, il sera à maintes reprises représenté, de l'objet bidimensionnel à la ronde-bosse, de la période prédynastique au crépuscule de l'ère pharaonique [10]. Car s'il est vrai que ce pachyderme peut être synonyme de calamité, il n'en demeure pas moins un animal ambivalent au statut théologique ambigu [11]. Ainsi, les hippopotames sont généralement pacifiques de nature, les femelles attentives encerclent les petits dans une attitude protectrice lors des déplacements [12] ; mais sitôt que survient un danger, ces paisibles herbivores - en particulier les mâles - se métamorphosent en bêtes agressives et colériques [13]. De fait, l'hippopotame présente dans la pensée égyptienne, et comme de nombreuses espèces du bestiaire égyptien, deux figures sexuellement différenciées,

[2] De l'égyptien kamehy : YoYotTe 2005, p. 249.

[3] Du grec hippopotamos : YoYoTte 2005, p. 248.

[4] Léon l'Africain, Description de l'Afrique, p. 446.

[5] Ibid., p. 447.

[6] Osborn \& Osbornová 1998, p. 144 ; De Jong 2001, p. 100-101; YоҮотте 2005, p. 249-250.

[7] Osborn \& Osbornová 1998, p. 144 ; Manlius 2000, p. 60.

[8] Manlius 2000, p. 62, fig. 1.

[9] YOYOTTE 2005, p. 249.

[10] Cf. Behrmann 1989. Pour les représentations en ronde-bosse de l'hippopotame datées de l'époque prédynastique, cf. HENDRICKX \& Depraetere 2010 ; Droux 2011.

[11] YoyotTe 2005, p. 249 ; Pirez 2012, p. 210.

[12] De Jong 2001, p. 100 ; YOYOTte 2005, p. 249-250.

[13] De Jong 2001, p. 100 ; YOYOTTE 2005, p. 249-250 et p. 260.

[14] Pirez 2012, p. 210.
I'une bienfaisante, I'autre malfaisante. Dans son aspect positif, I'hippopotame, et plus spécifiquement la femelle, incarne une force protectrice et maternelle [14], agent de résurrection [15] : ce sont les figurines d'hippopotame en faïence du Moyen Empire, décorées de motifs aquatiques et végétaux dépeignant son milieu de vie [16]. En raison de cet aspect protecteur, plusieurs déesses [17] prennent la forme d'un hippopotame, parmi lesquelles Thouéris, une divinité composite : tête et corps d'hippopotame, queue de crocodile, mains et pieds de lionne [18]. Vénérée dans la sphère domestique pour la protection des femmes enceintes et des nouveau-nés, elle a le ventre rond d'une femme sur le point d'accoucher et les seins lourds d'une mère allaitante. Ses origines pourraient remonter à l'époque prédynastique [19]. Autre déesse pacifique, la dénommée Hedjet, I'Hippopotame Blanche, est l'actrice principale du $\mathrm{Hb} \mathbf{H}$ d.t la « Fête de la Blanche » [20]. Attesté sur des fragments de bas-reliefs de I'Ancien Empire jusqu'à la période tardive, ce festival met en scène la visite du roi, coiffé de la couronne rouge de Basse-Égypte, à Hedjet, hippopotame blanche reposant paisiblement de ses quatre pattes sur un traîneau [21]. À I'opposé, dans son aspect négatif, I'hippopotame, et particulièrement le mâle, est craint en raison de son côté destructeur et féroce. Les figurines en argile d'hippopotames recouvertes d'engobe rouge évoquent certainement le caractère négatif de l'animal [22]. C'est en raison de cet aspect destructeur que I'hippopotame deviendra à partir de l'époque ramesside I'une des apparences de Seth [23].

Au vu de son image négative et du préjudice qu'il cause au royaume en ravageant les cultures, il n'est pas étonnant que les anciens Égyptiens se soient livrés à une chasse à

[15] VeRNUS 1991, p. 337

[16] À moins que ces statuettes thériomorphes ne représentent les deux aspects, positif et négatif, du pachyderme : LACOVARA, 1992, p. 24. Voir également Yoyotre 2005, p. 259.

[17] Thouéris, Ipet, Reret, Hedjet. Houser-Wegner 2001, p. 351 ; PIREZ 2012.

[18] Gundlach 1986 ; Houser-Wegner 2001 ; Pirez 2012.

[19] C'est ce que suggèrerait un peigne en ivoire représentant deux hippopotames dos à dos, dressés sur leurs pattes arrière, le ventre rebondi, caractéristiques de Thouéris. Toutefois son authenticité reste douteuse : I'objet provient du marché des antiquités et ne possède aucun parallèle. Cf. Seligman 1916, p. 53.

[20] YOYOTTE 2005, p. 260.

[21] SäVE-SÖDERBERGH 1953, p. 47-55 ; De WITt 1958 ; KAISER 1988 ; LACOVARA 1992 ; Altenmüller 1994 ; Kaiser 1997 ; Yoyotte 2005, p. 259-260.

[22] LACOVARA 1992, p. 23, 24.

[23] YOYOTTE 2005, p. 261. 
I'hippopotame, réelle ou fictive. En dehors de son caractère défensif, la traque du cheval de fleuve apportait par ailleurs des ressources variées : défenses, peau, graisse, excréments et chair [24]. Depuis la préhistoire, les techniques de chasse n'ont guère évolué, en atteste l'iconographie des chapelles funéraires et des temples durant toute la période pharaonique [25]. Une pratique quasiment identique a été filmée par le réalisateur Jean Rouch dans un documentaire ethnographique tourné de janvier à juin 1951 sur le fleuve Niger, Bataille sur le grand fleuve. Les chasseurs, à bord d'embarcations sommaires, abordent la bande d'hippopotames et repèrent leur proie. Ils sont armés de harpons constitués d'un manche en bois terminé par une barbelure amovible, attachée à une corde, elle-même reliée à un flotteur. Lorsque la tête du harpon se fiche dans l'animal, elle se détache du manche mais reste solidaire de la corde maintenue par le chasseur. Chaque fois que la bête sort la tête pour respirer, les coups pleuvent. Les hommes tirent de préférence en direction de la gueule du pachyderme. Ils tendent les cordes, forçant la créature enragée à tourner la tête vers l'embarcation et l'empêchent de plonger pour s'enfuir [26]. Avec parfois plus de cinquante harpons lui transperçant la peau, I'hippopotame s'abandonne à son dernier combat. Mort d'épuisement, il est amené jusqu'au rivage par les harponneurs, qui le suivent à la trace grâce aux flotteurs [27]. Seule différence entre les anciens Égyptiens et les hommes filmés par Jean Rouch au xx siècle : l'imagerie égyptienne [28] ne figure généralement qu'un seul harponneur aux prises avec un hippopotame. Debout sur une barque, il tient d'un bras levé un harpon, qu'il s'apprête à lancer sur l'animal ; de l'autre, il empoigne le rouleau des cordes déjà fichées dans la peau du colosse. Distraction ou acte défensif lorsqu'elle est perpétrée par de simples mortels sur les murs des tombes de I'Ancien Empire et du Nouvel Empire [29], la mise à mort de l'hippopotame est acte rituel lorsqu'elle est accomplie par des divinités comme Horus [30] ou Hémen [31] ou par le monarque [32]. Précisément, jusqu'où peut-on remonter la piste de cette chasse royale ritualisée ? Avec pour point de départ des sources tex-

[24] Ibid., p. 251-252.

[25] Vandier 1964, p. 776-786; Behrmann 1989.

[26] SÄVE-SÖDERBERGH 1953, p. 1-15; YoYOtTE 2005, p. 252.

[27] YOYOTTE 2005, p. 252.

[28] Cf. SÄve-Söderbergh 1953 ; Behrmann 1989. L'iconographie de I'Ancien Empire fait néanmoins exception et présente généralement plusieurs individus attelés au harponnage.

[29] SÄve-SöderberGH 1953 ; BeHRMANN 1989.

[30] Papyrus du Caire no 86637 : BAKIR 1966, pl. VII 4-VIII 3 ; BEHRMANN 1989, doc. 185b.1; Papyrus Sallier IV 2,6-3,5 : Behrmann 1989, doc. 185b.2 ; Papyrus Chester Beatty No1 : Broze 1996, p. 74-79; temple d'Edfou : LABRIQUE 1993.

[31] Textes des Pyramides : Pyr. 235a-b ; tombe d'Ânkhtyfy : VANDIER 1946 ; SÄVE-SöderberGH 1953, p. 29-33; VANDIER 1964, p. 781-783 tuelles et iconographiques relatives au règne de Den, dont la pierre de Palerme, I'enquête se poursuivra avec I'examen du matériel iconographique et archéologique du Prédynastique, qui permettra de mieux comprendre I'origine de ce rite et le rapport entre l'indice iconographique et la réalité historique de la pratique rituelle. Enfin l'étude du corpus dynastique permettra de révéler le rôle joué par ce rite cynégétique dans la construction de l'idéologie pharaonique.

\section{« TRANSPERCER L'HIPPOPOTAME »: AUTOPSIE D'UNE ENTRÉE DE LA PIERRE DE PALERME}

La pierre de Palerme [33], plus ancienne liste royale égyptienne, n'est en réalité qu'un des fragments - le plus imposant - du monument qui portait initialement les annales de la période prédynastique à la $V^{e}$ dynastie. La pierre, inscrite à l'origine sur les deux faces, recense les principaux événements qui se seraient déroulés au cours des cinq premières dynasties. Les inscriptions sont disposées en registres, qui comprennent chacun une série de compartiments rectangulaires. Les traits délimitant les compartiments figurent en fait le signe de l'année, rnp.t, qui précède l'inscription: chaque case équivaut ainsi à une année de règne [34] ; le nom du roi régnant est inscrit au-dessus des registres. La partie inférieure de chacune des cases enregistre vraisemblablement les hauteurs annuelles de la crue du Nil [35]. La date de compilation des annales reste toujours débattue; cependant, on propose généralement l'époque à laquelle la liste prend fin, soit la Ve dynastie [36].

L'entrée des annales qui nous occupe est située à la huitième colonne du troisième registre et attribuable au règne de Den [37], cinquième roi de la Ire dynastie (fig. 1). Elle est encadrée de compartiments relatant des événements typiques des annales: la visite de temples [38], la création d'images divines [39], la course du taureau Apis [40] ou encore la célébration de festivals [41].

[32] Cf. Behrmann 1989.

[33] Musée archéologique Salinas, Palerme. Pour une synthèse sur la pierre de Palerme et les fragments associés, cf WiLkinson 2000.

[34] WiLKINSON 2000, p. 19.

[35] Ibid., p. 20.

[36] Ibid., p. 23-24.

[37] Pour I'attribution du troisième registre au règne de Den, cf. WiLKINSON 2000, p. 103-105.

[38] Registre III, entrées 1 et 9.

[39] Registre III, entrées 11,13 et 14.

[40] Registre III, entrée 12.

[41] Registre III, entrées 3, 5 et 6. 


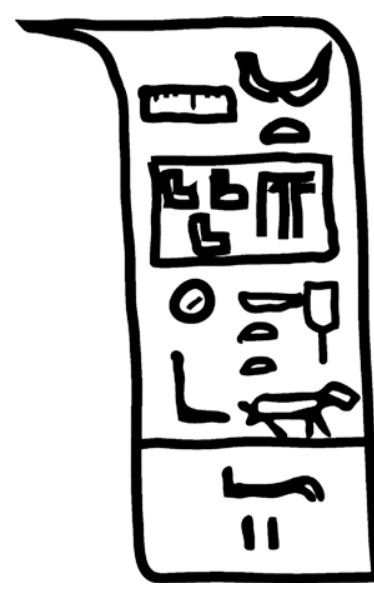

Figure 1 : colonne 8 de la rangée 3 de la pierre de Palerme. Dessin de l'auteure.

\section{Traduction et commentaire de l'entrée}

La huitième entrée du troisième registre consigne deux événements du règne de Den (fig. 1). Depuis son acquisition vers 1859 par l'italien Fernandino Gaudiano - ou par son géniteur [42] -, la pierre de Palerme a fait couler I'encre d'une pléiade d'auteurs, dont la plupart ont entrepris de traduire et commenter l'œuvre [43]. Rien d'étonnant donc à ce que l'inscription en question ait été translittérée et traduite à plusieurs reprises, avec de légères divergences au gré des plumes (tab. 1).
La première partie de l'énoncé, qui se rapporte à l'inauguration d'un lac rattaché à un édifice cultuel, ne donne lieu à aucune divergence de traduction et ne sera pas commentée plus avant. La seconde partie ne semble de premier abord pas non plus être sujette à des différences majeures de lecture : tous les traducteurs lisent l'inscription «transpercer l'hippopotame ». On évoquera tout de même le fait que l'hippopotame a été diversement translittéré selon les auteurs par $d b$ [44] ou $h_{3} b$ [45], ses deux principaux noms dans la langue égyptienne. J. Ogdon comprend par ailleurs les deux signes généralement lus en tant que phonogrammes de $h(3) b$ comme un substantif à part entière, qu'il lit Nekheb. Quant au verbe, tous s'accordent pour y voir l'infinitif de sti « abattre », «transpercer», «harponner » [46]. On déclare même que cet énoncé ne pose aucun problème de traduction : ainsi T. Wilkinson écrit «The spelling of the word stt 'spearing' is clear and unambiguous »[47], tandis que G. Godron affirme « La dernière phrase n'offre aucune difficulté » [48].

Pourtant, peut-on vraiment affirmer sans autre discussion que le premier signe, idéogramme supposé de lo Th $^{\circ}$ sti, « abattre», figure bien le signe F29 To, c'est-à-dire une peau de bovin percée d'une flèche ?

Tableau 1 : translittérations et traductions proposées par les différents auteurs pour l'entrée 8, registre 3 de la pierre de Palerme.

\begin{tabular}{|c|c|c|}
\hline \multirow[t]{4}{*}{ TRANSLITTÉRATION } & TRADUCTION & BIBLIOGRAPHIE \\
\hline & $\begin{array}{l}\text { «Öffnen (?) des SeesvomHause 'Sitz } \\
\text { der Götter'. Schiefsen des Nilpferdes » }\end{array}$ & SCHÄFER 1902, p. 20. \\
\hline & $\begin{array}{l}\text { «Opening of the Lake of the House } \\
\text { (called) : 'Thrones of the Gods'. } \\
\text { Shooting of the hippopotamus » }\end{array}$ & BREASTED 1906, p. 60. \\
\hline & $\begin{array}{l}\text { «Opening of the lake of the House Isut- } \\
\text { Neteru. Shooting of the hippopotamus » }\end{array}$ & $\begin{array}{l}\text { NEWBERRY \& WAINWRIGHT } \\
1914 \text {, p. } 152 .\end{array}$ \\
\hline Wpt $H r$ (?) Ht-Ntrw-İswt sp stt $d b$ & $\begin{array}{l}\text { «Opening ( ?) of the House of the } \\
\text { Thrones of the Gods. Occasion ( ?) of } \\
\text { shooting the hippopotamus» }\end{array}$ & PATON 1915 , tableau $n^{\circ} 2$. \\
\hline \multirow[t]{2}{*}{ Wp.t š st.w-ntr.w sttt (?) db(i) Nhbb (?) } & $\begin{array}{l}\text { «Opening of the lake (of) 'thrones of } \\
\text { the gods' (with the) shooting of ( ?) the } \\
\text { hippopotamus (in) Nekheb ( ?) » }\end{array}$ & OGDON 1982, p. $42-43$ \\
\hline & $\begin{array}{l}\text { «Ouvrir (= inaugurer) le lac de } \\
\text { (I'édifice) Hauteur-des-dieux. Tirer } \\
\text { (=chasser) I'hippopotame » }\end{array}$ & GODRON 1990, p. 129. \\
\hline Wpt-š swt-ntrw stt h̆(3)b & $\begin{array}{l}\text { «Opening the (sacred) lake (at the } \\
\text { building) 'thrones of the gods'; } \\
\text { spearing the hippopotamus » }\end{array}$ & WILKINSON 2000, p. 112. \\
\hline
\end{tabular}

[42] WiLKINSON 2000, p. 20.

[43] Sur I'histoire de la recherche sur la pierre de Palerme, cf. WiLKINSON 2000 , p. $28-45$.

[44] Wb. V, 433.14-17.
[45] $W b$. III, 229.5-6

[46] Wb. IV, 326.2-14; AnLex. 77.3948, 78.3913, 79.2824.

[47] WiLKINSON 2000, p. 114.

[48] GodRon 1990, p. 128. 
Et que dire de I'hippopotame, qui semble manifestement manquer de son caractère le plus emblématique : son ventre proéminent ? Assurément, pour être validée, la traduction demande une investigation plus poussée dans la documentation épigraphique égyptienne. Débutons avec le verbe 10 tơ sti : il s'agit tout d'abord de vérifier s'il existe d'autres attestations de la graphie tro figurée sur la pierre de Palerme. Premier point d'importance, le verbe est attesté dès I'Ancien Empire [49] ; mieux : il est habituel de l'écrire à cette époque en omettant l'unilitère $s$ Tfo $0[50]$. Deuxième point, il est fréquent de rencontrer sur les inscriptions sa forme infinitive, où la peau transpercée côtoie les deux petits pains $T^{\circ}$ [51]. Ainsi, la graphie du verbe sur la pierre de Palerme paraît conforme ; mais que dire du premier hiéroglyphe, qui n'évoque à priori pas clairement le signe F29 Tlusieurs inscriptions semblent attester que le signe de la peau de bovin percée d'une flèche pouvait avoir une forme proche du hiéroglyphe gravé sur la pierre de Palerme, particulièrement durant l'Ancien Empire (tab. 2).

C'est le cas de la tombe numérotée trois [52] à Béni Hassan, décorée d'une fresque sur laquelle le défunt, debout sur une barque, harponne deux poissons. La faune aquatique est représentée alentours sous la forme d'un crocodile, de divers poissons et d'un hippopotame, la gueule ouverte. Dans le texte accompagnant l'image, I'une des activités effectuées par le défunt consiste à transpercer I'hippopotame, st.t h3b (tab. 2, n²). Ici la flèche fichée dans la peau du signe F29 ne ressort pas du côté opposé. Seule la partie arrière, munie d'un empennage, est visible : le signe se rapproche donc, sans être exactement semblable, de celui gravé dans les annales. De même, dans la tombe de Mérérouka [53] à Saqqara, contemporaine de la VI ${ }^{e}$ dynastie, la légende qui complète la scène de harponnage de plusieurs pachydermes, débute par st.t ta (tab. 2, n³). Là encore, la flèche reste fichée dans la peau et ne la transperce pas. Même constat dans la tombe de Kaiemânkh, à Giza, également datée de la VIe dynastie (tab. 2, n4) [54] ainsi que dans plusieurs formules des Textes des Pyramides [55]. Plus éclairantes encore sont les inscriptions gravées dans la tombe d'Idou [56] à Dendera, remontant elles aussi à la $V{ }^{e}$ dynastie (tab. 2, n5). Non seulement la flèche ne traverse pas la peau animale, mais l'empennage est à peine visible, donnant à l'instrument la forme d'un rectangle allongé. De plus, la partie inférieure de la peau, au lieu de présenter l'aspect habituel d'une goutte étirée, est rectangulaire et anguleuse. Dans l'ensemble, le signe F29 reproduit dans la tombe d'Idou offre une silhouette assez ressemblante à celle du hiéroglyphe de la pierre de Palerme. Ainsi, les différents parallèles tendent à montrer que la première partie de l'énoncé transcrit effectivement l'infinitif du verbe 10 个o sti, «transpercer». Qu'en estil du second terme de la phrase?

Si les auteurs sont partagés entre les lectures

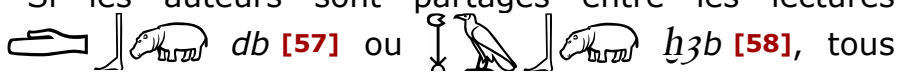

Tableau 2 : paléographie du signe

\begin{tabular}{|c|c|c|c|c|}
\hline 1 & & $\begin{array}{l}\text { Pierre de Palerme, } \\
\text { colonne } 8 \text {, rangée } 3 .\end{array}$ & $V^{e}$ dynastie & Dessin de l'auteure. \\
\hline 2 & & $\begin{array}{l}\text { Tombe } n^{\circ} 3 \text { de Béni } \\
\text { Hassan. }\end{array}$ & XII dynastie & $\begin{array}{l}\text { D'après NeWBERRY 1893, } \\
\text { pl. XXXIV. }\end{array}$ \\
\hline 3 & $\sqrt{0}$ & $\begin{array}{l}\text { Tombe de Mérérouka, } \\
\text { Saqqara. }\end{array}$ & $\mathrm{VI}^{\mathrm{e}}$ dynastie & $\begin{array}{l}\text { D'après BeHRMANN 1989, } \\
\text { doc. 92a. }\end{array}$ \\
\hline 4 & $8 \rrbracket$ & $\begin{array}{l}\text { Tombe de Kaiemânkh, } \\
\text { Giza. }\end{array}$ & $\mathrm{VI}^{\mathrm{e}}$ dynastie & $\begin{array}{l}\text { D'après JUNKER 1940, } \\
\text { fig. } 8 .\end{array}$ \\
\hline 5 & (7) 0 & $\begin{array}{l}\text { Tombe d'Idou, } \\
\text { Denderah }\end{array}$ & $\mathrm{VI}^{\mathrm{e}}$ dynastie & D'après Petrie 1900a, pl. V. \\
\hline
\end{tabular}

[49] Wb. IV, 326.

[50] Ibid.

[51] Säve-Söderbergh 1953, fig. 4 ; Behrmann 1989, doc. 92a, 97, $125 \mathrm{c}, 129,161 \mathrm{a}, 161 \mathrm{e}, 161 \mathrm{~g}$.

[52] Newberry 1893, pl. XXXIV ; Behrmann 1989, doc. 125c.

[53] Behrmann 1989, doc. 92a.
[54] Ibid., doc. 97.

[55] Pyr. 120a, 123f, 1197d, 1781d, 1863a, 2209b. Cf. AlLen 2013.

[56] Petrie 1900a, pl. v.

[57] $W b . V, 433.14-17$.

[58] Wb. III, 229.5-6. 
s'accordent donc à traduire la suite de l'énoncé par « hippopotame ». À choisir entre les deux substantifs, on penchera plutôt pour une lecture $h_{3} b$ : en effet le signe rond qui précède la jambe paraît plutôt correspondre à I'unilitère Aa1 $\ominus$ qu'à la main $ح$ [59]. On aurait ainsi une orthographe du substantif $\theta$ Fresod $h(3) b$, graphie qui n'est pas inhabituelle dans la documentation de I'Ancien Empire [60]. Mais le déterminatif au corps si chétif représente-t-il effectivement un hippopotame ? C'est ce que tendent à montrer deux scènes [61] de la tombe de Ttí, datée des $\mathrm{V}^{\mathrm{e}}-\mathrm{VI}^{\mathrm{e}}$ dynasties, sur lesquelles apparaît le nom db.t, déterminé par un pachyderme au corps tout aussi grêle que celui de l'animal figuré sur la pierre de Palerme (fig. 2).

Si les lectures st.t et $h(3) b$ sont confirmées par les différents parallèles, l'expression st.t $\underline{h}(3) b$ elle-même est

Figure 2 : panneaux de la tombe de Teti, Giza, $V^{e}-V^{e}$ dynasties. Le premier hippopotame se situe sur l'image supérieure, au bout de la première ligne à droite ; le second se situe sur l'image inférieure, en fin de sixième colonne. D'après LePsius 1842, pl. VIIla.
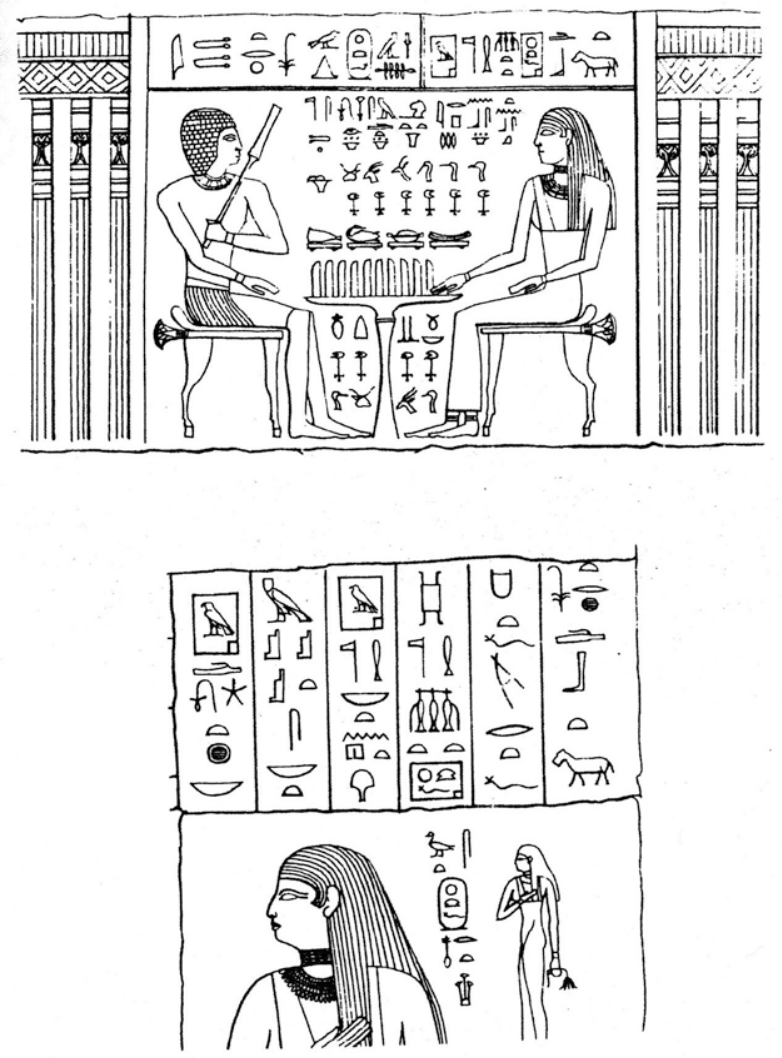

[59] On rappellera toutefois que J. Ogdon (OGDON 1982, p. 42-43) identifie les deux premiers signes à la ville de Nekheb et comprend le signe de l'hippopotame comme un idéogramme pour rendre le mot $d b$. Or, une telle graphie de Nekheb - le signe de la ville 049 suivi de la jambe - ne semble pas exister ailleurs ( $W b$. II, 309.2). D. Paton (PATON 1915 , tableau $n^{\circ} 2$ ) interprète pour sa part le signe circulaire comme I'idéogramme $s p \mathrm{O} 50$; si I'on suit cette hypothèse, I'hippopotame serait transcrit uniquement par I'unilitère $b$ suivi du déterminatif sous la forme dudit animal : or, une telle graphie n'a aucun parallèle connu, que I'on choisisse la translittération $d b$ ou la translittération $h_{3} b$.

[60] Wb. III, 229.5. alors tout à fait vraisemblable. En effet le verbe st.t est le terme habituellement employé pour parler d'une chasse à I'hippopotame au harpon [62]. L'expression apparaît d'ailleurs dans plusieurs tombes privées [63].

En somme, la lecture tro $\theta$ ented st.t $h(3) b$ «transpercer I'hippopotame » est plausible graphiquement et sémantiquement ; néanmoins, d'autres sources contemporaines du règne de Den permettent-elles d'appuyer cette traduction?

\section{Documents contemporains du règne de Den}

Une incursion dans la riche documentation iconographique produite durant le règne de Den révèle le dynamisme de la figure royale dès la période thinite. Pharaon est engagé dans diverses activités rituelles, et la mise à mort d'un hippopotame semble bien en faire partie. Ainsi, sur plusieurs étiquettes d'année - petite tablette rectangulaire qui recenserait les événements marquants d'une année de règne - le roi est figuré main levée en arrière, harpon brandi en direction de l'eau [64] (tab. 3). Sur l'une d'entre elles, I'hippopotame est déjà à la merci du souverain, avec plusieurs harpons fichés dans son dos (tab. 3, étiquette $\mathrm{n}^{\circ} 1$; fig. 3) ; sur une autre, Den est

Figure 3 : étiquette au nom du roi Den. La chasse à l'hippopotame se situe dans la quatrième colonne. D'après GodRoN 1990, pl. III.6.

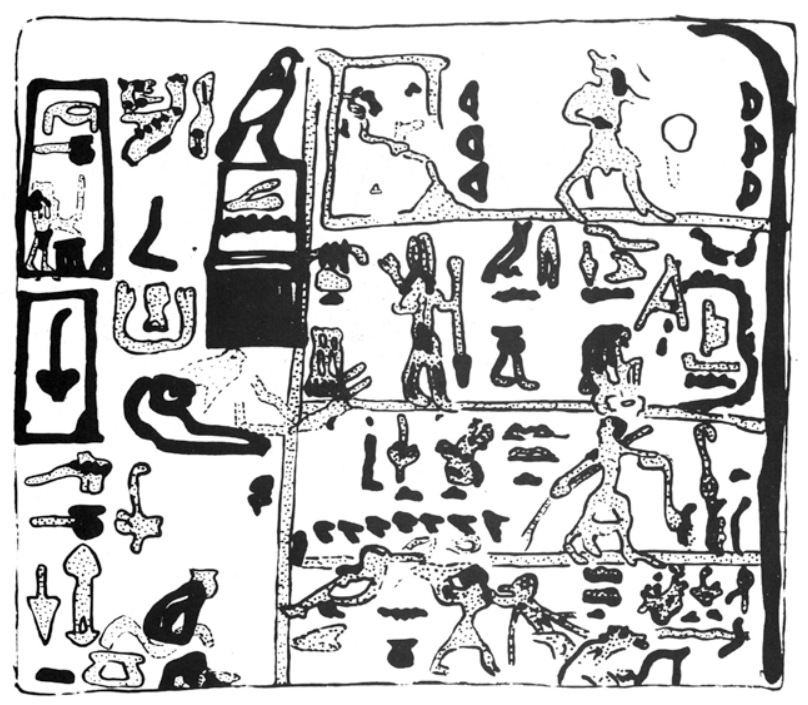

[61] BeHRMANn 1989, doc. 105b.

[62] $W b$. IV, 326.10.

[63] Tombe $\mathrm{n}^{\circ} 3$ de Béni Hassan, XII e dynastie (NewberRy 1893, pl. XXXIV) ; Tombe de Neb-seni, Cheikh Abd al-Gourna, XVIII dynastie (?) (Behrmann 1989, doc. 161g); Tombe de Douaouneheh, TT 125, Cheikh Abd al-Gourna, XVIII e dynastie (ChAMPOLLION 1844, p. 515) ; Säve-Söderbergh 1953, fig. 4.

[64] Il est possible que deux étiquettes supplémentaires se rapportent à une chasse à I'hippopotame: Petrie 1902, pl. XI, fig. 8 ; DreYER et al. 1998, pl. $12 f$. 
Tableau 3 : sources datées du règne de Den figurant une chasse à l'hippopotame.

\begin{tabular}{|c|c|c|c|c|}
\hline SOURCE Nº & TYPE & SITE & $\begin{array}{c}\text { LIEU DE } \\
\text { CONSERVATION }\end{array}$ & BIBLIOGRAPHIE \\
\hline 1 & Étiquette & $\begin{array}{l}\text { Abydos, Umm el-Qaab, } \\
\text { tombe } 83 \text { au sud-ouest } \\
\text { du tombeau de Djer }\end{array}$ & $\begin{array}{l}\text { Paris, Musée du } \\
\text { Louvre, } 25.268\end{array}$ & GodRON 1990, pl. I à III. \\
\hline 2 & Étiquette & $\begin{array}{l}\text { Abydos, Umm el Qaab, } \\
\text { cimetière de Den }\end{array}$ & Inconnu & Petrie 1900, pl. XIV, fig. 8. \\
\hline 3 & Étiquette & $\begin{array}{l}\text { Abydos, Umm el Qaab, } \\
\text { cimetière de Den }\end{array}$ & $\begin{array}{l}\text { Abydos, SCA Storage } \\
\text { Magazine, Ab K } 2503\end{array}$ & DREYER et al. 1998, pl. 12d. \\
\hline 4 & Scellé & $\begin{array}{l}\text { Abydos, Umm el Qaab, } \\
\text { cimetière de Den }\end{array}$ & Inconnu & $\begin{array}{l}\text { PetRIE } 1901 \text {, pl. 7, fig. 5-6 ; } \\
\text { KAPLONY 1963, III, fig. } 364 \text {. }\end{array}$ \\
\hline 5 & Scellé & $\begin{array}{l}\text { Abydos, Umm el Qaab, } \\
\text { cimetière de Den }\end{array}$ & Inconnu & $\begin{array}{l}\text { Petrie } 1900 \mathrm{~b}, \mathrm{pl} .32 \text {, fig. } 39 \text {; } \\
\text { KaPLONy 1963, III, fig. } 365 \text {. }\end{array}$ \\
\hline 6 & Scellé & $\begin{array}{l}\text { Abydos, Umm el Qaab, } \\
\text { cimetière de Den }\end{array}$ & $\begin{array}{c}\text { Ab K } 6500 \text { et } 6501 \\
\text { (DAI Cairo) }\end{array}$ & MüLLeR 2008, fig. 3. \\
\hline 7 & Scellé & $\begin{array}{l}\text { Abydos, Umm el Qaab, } \\
\text { cimetière de Den }\end{array}$ & & MÜLLER 2014. \\
\hline
\end{tabular}

représenté à bord d'une embarcation, probablement une barque (tab. 3 , étiquette $n^{\circ} 3$ ). Signalons par ailleurs que sur la seule tablette où la tête du souverain est visible de manière nette, il est coiffé de la couronne rouge de Basse-Égypte (tab. 3, étiquette $n^{\circ} 3$ ).

Une seconde catégorie de sources témoigne de la mise à mort de I'hippopotame par le roi Den : les scellés (tab. 3). Quatre sceaux ont pu être reconstitués [65] ; tous font partie des Festsiegel [66] et figurent le souverain harponneur. Le roi y est représenté selon la figure stéréotypée que I'on retrouve plus tard dans l'iconographie pharaonique : d'une main levée il tient son harpon, qu'il s'apprête à abattre sur l'animal ; de l'autre, il maintient l'hippopotame hors de l'eau grâce aux rouleaux de cordes reliés aux barbelures qui transpercent la peau du pachyderme. Deux des empreintes affichent une seconde scène de chasse tout à fait remarquable : le roi, en corps à corps cette fois, combat à mains nues l'animal, dressé sur ses pattes arrière [67] (tab. 3, scellés $n^{\circ} 4$ et 7 , fig. 4). Cette image, unique dans l'iconographie égyptienne, révèle la dimension héroïque et la part fictive de ces représentations royales [68]. Elle n'est pas non plus sans rappeler la figure du Maître des Animaux, à peine plus ancienne, où un individu retient par sa force deux animaux antithétiques [69].

[65] Cf. tab. 3. Un cinquième sceau, fragmentaire, représente le roi harponnant un animal. Malheureusement, on ignore s'il s'agit d'un hippopotame ou d'un poisson : DreYer et al. 2011, p. 69-71, fig. 23.

[66] MüLLER 2008, p. 480, n. 5. On trouvera des illustrations de ces scellés dans Petrie 1900b, pl. 32, fig. 38-39; KAPLONY 1963, III, fig. 11, 237, 364, 365, 1032 ; KAPLONY 1963, I, p. 129 ; MüLLER 2008, fig. 3 ; DREYER et al. 2011, fig. 23.

[67] L'identification de I'animal à un hippopotame est confirmée par la découverte de nouveaux fragments (communication personnelle de V. Müller)
À nouveau, le roi harponneur est coiffé de la couronne rouge. Représentées sur une ligne qui pourrait rappeler une base, il est possible que ces images soient des reproductions de statues royales [70]. Ces scènes cynégétiques sont accompagnées d'autres décors : sur deux scellés (tab. 3 , scellés $n^{\circ} 4$ et 5 ), Den, un pied en avant et la tête ceinte de la couronne blanche de Haute-Égypte, tient d'une main un bâton et de l'autre un sceptre. La troisième empreinte [71] montre des captifs, bras liés derrière le dos, tête décapitée exposée entre leurs jambes, une image déjà visible sur la célèbre palette de Narmer (tab. 3, scellé $n^{\circ} 6$, fig. 5). L'association de prisonniers à la chasse de l'hippopotame n'est certainement pas

Figure 4 : scellé au nom du roi Den. D'après KAPLONY 1963, vol. III, Fig. 364

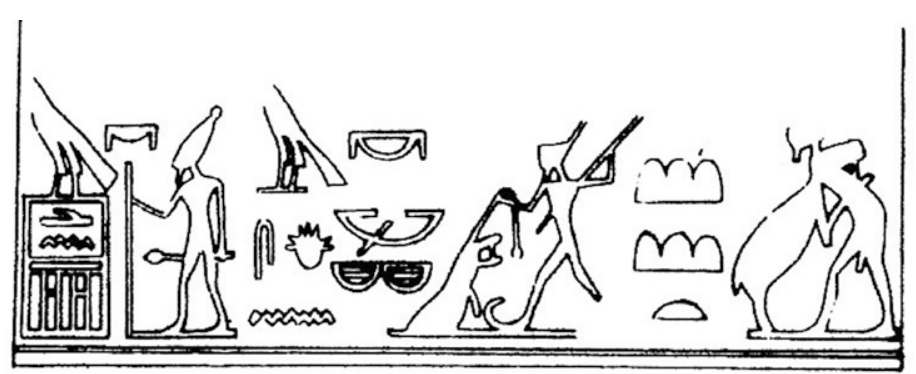

[68] YoYOTTE 2005, p. 250.

[69] Tombe 100, Hiérakonplis: Quibell \& GREen 1902, pl. LXXVI ; manche de couteau du Gebel el-Arak : Czichon \& SieverTSEn 1993, fig. 1 ; ivoire décoré, Hiérakonpolis : VANDIER 1952, p. 551.

[70] Vernus 1991, p. 335 ; Yoyotte 2005, p. 250. Cf. MüLler 2008, p. 487 pour un avis contraire.

[71] MüLLER 2008, fig. 3. De nouveaux fragments de scellés découverts attestent que l'animal harponné est bien un hippopotame : MüLLER 2014. 
anodine : les deux scènes doivent probablement être comprises comme l'expression de la domination sur les forces chaotiques issues des mondes animalier et humain [72].

En outre, sur trois empreintes de sceaux, la légende qui accompagne l'image du roi chasseur mentionne l'expression Hrw-msnw, « Horus le harponneur » [73]. La figure royale chasseresse est donc identifiée dès la période thinite à Horus. L'Horus maître de Mesen ou Horus le harponneur n'a pas de lieu de culte attitré, c'est pourquoi il existe un Mesen de l'est, de l'ouest et de la partie centrale [74]. Dans un titre du début de I'Ancien Empire, il est rattaché à la ville de Bouto en Basse-Égypte [75]. Faut-il y voir un lien avec le port systématique de la couronne rouge de Basse-Égypte par le roi sur les scènes de chasse à I'hippopotame ?

Ainsi, les parallèles extraits du corpus épigraphique renforce la lecture «transpercer I'hippopotame», proposée pour l'entrée 8 du règne de Den. La traduction de ce passage de la pierre de Palerme semble par ailleurs appuyée par les nombreuses sources contemporaines figurant le roi en train de chasser l'animal. La profusion des monuments narrant l'épisode témoigne assurément de son importance primordiale durant le règne de Den. Les légendes qui accompagnent les représentations du souverain harponneur ont révélé que, dès l'époque thinite, cette image royale est identifiée à Horus. Elles mettent également en avant l'association de la mise à mort de l'hippopotame avec les ennemis ligotés et décapités. La chasse de I'hippopotame constitue ainsi - sinon une activité réelle - du moins un thème iconographique fort, récurrent et bien implanté durant le règne de Den. De fait, cet ancrage révèle sans doute une origine plus ancienne encore, qu'il faut découvrir en remontant la piste préhistorique.

\section{LA CHASSE À L'HIPPOPOTAME, UNE PRATIQUE RITUELLE ATTESTÉE DĖS LE PRÉDYNASTIQUE...}

Le Prédynastique est certainement la période qui a fourni la documentation la plus prolifique de toute I'histoire égyptienne sur l'iconographie de I'hippopotame, et en particulier sa chasse. Ainsi les vases de type White Cross-lined, déposés dans les tombes de Nagada I à Nagada IIA [76], foisonnent d'hippopotames chassés au harpon, peints dans une couleur blanche contrastant avec l'engobe rouge brique de la céramique (fig. 6) [77]. Fiché dans le corps de l'animal, le harpon prend la forme d'un trait doublé d'une ligne ondulée, qui représentent respectivement le manche et la corde qui y est enroulée. Le rouleau de corde restante est souvent représenté à l'extrémité par des cercles concentriques ; Iorsque l'artiste a choisi de dépeindre le chasseur - ce qui n'est pas toujours le cas [78] - celui-ci tient parfois le rouleau. Sur deux poteries [79] aux décors remarquables, la traque de I'hippopotame côtoie deux thématiques fort intéressantes : d'une part une chasse, ici un chien aux trousses d'un ibex et d'un oryx [80], là un bovin aux longues cornes [81] ; d'autre part des individus qui semblent liés les uns aux autres, sans doute des captifs [82] (fig. 7 et 8). L'association entre la chasse d'un hippopotame

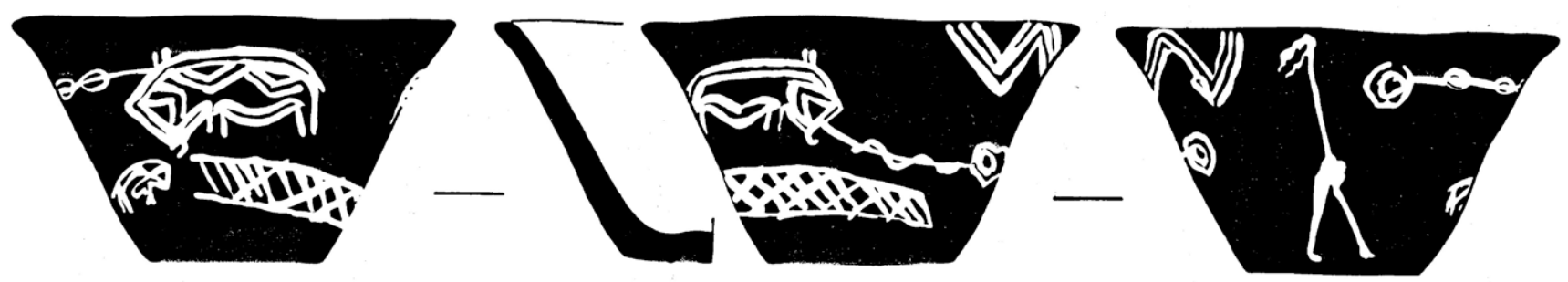

[72] MÜLLER 2008.

[73] Cf. KAPLONY 1963, II, p. 1142 : « Horus der Harpunierer aus Gold, Schiff der beiden Herrinnen (Herren) »; MüLLER 2008, p. 480-482 : Hrw-msnw «Horus der Harpunierer» et Pj-Hrwmsnw «Sitz von Horus, dem Harpunierer ». Le mot msn désigne le flotteur relié au harpon par la corde; par extension msnw nomme les harponneurs, les individus qui participent à la chasse à I'hippopotame. Cette forme d'Horus apparaît également dans le mythe fondateur d'Horus et Seth : avec son acolyte Horus de Behedet, il transperce son rival Seth, qui a pris I'apparence d'un hippopotame (VERNUS 1982).

[74] Vernus 1982, col. 108.

[75] Ibid.
[76] HENDRICKX 2006.

[77] GrAFF 2009, n० 40, 62, 63, 74, 77, 84, 86, 98, 117, 158, 161, 162.

[78] Il semble que seule la présence de I'arme, c'est-à-dire le harpon, peut suffire à rendre l'activité cynégétique : GRAFF 2009, n 40,62, $63,74,77$.

[79] Tombe U-415, Umm el-Qaab, Abydos : DreYer et al., 2003, p. 81, fig. 6a, GrafF 2009, n० 162 ; Tombe U-415, Umm el-Qaab, Abydos : DreYER et al., 2003, p. 81, fig. 5, GrafF 2009, n 161.

[80] GRAFF 2009, n० 162.

[81] Ibid., n० 161.

[82] HENDRICKX 1998. 

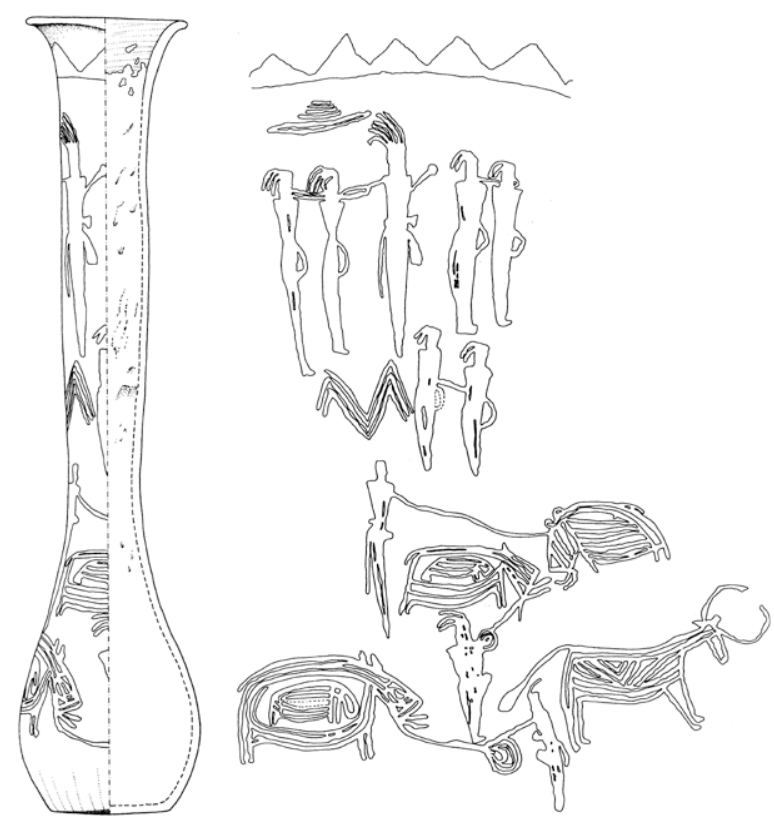

Figure 7 : vase White Cross-lined, Abydos, tombe U-415. D'après DREYER et al. 2003, Fig. 5.

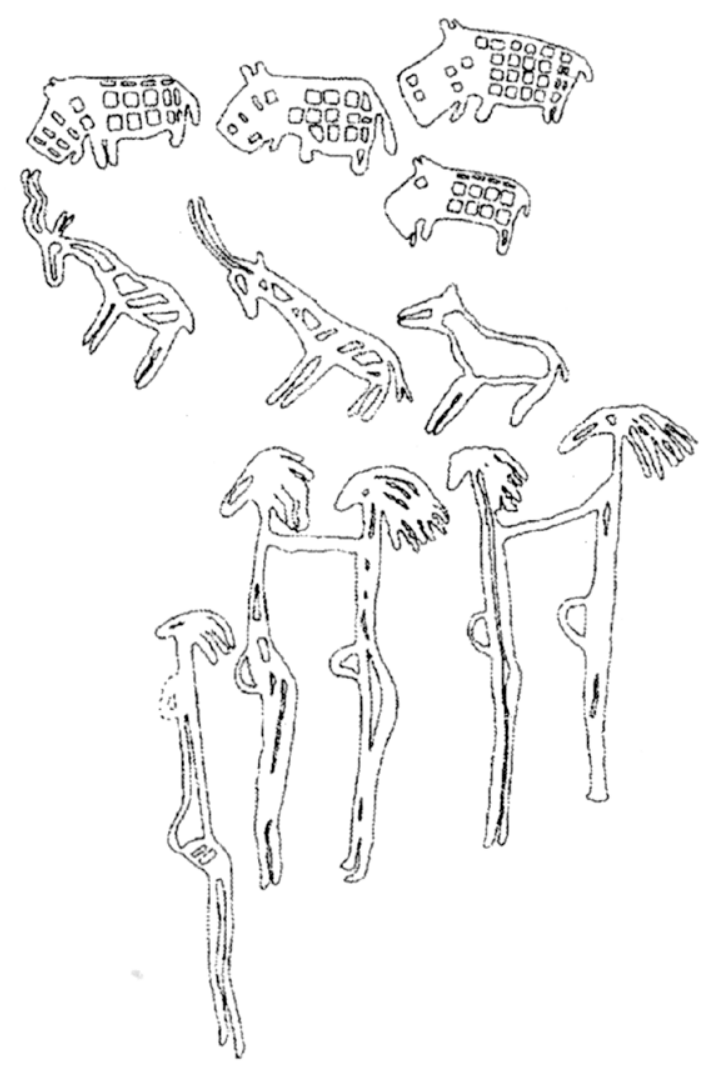

Figure 8 : vase White Cross-lined, Abydos, tombe U-415. D'après HARTUNG 2010, Fig. 4c.

[83] HENDRICKX \& EYCKERMAN 2012, p. 28-32. Pour une interprétation différente des scènes dépeintes sur les deux vases de la tombe U-415, cf. infra.

[84] HARTUNG 2010, p. 110-111.

[85] Behrmann 1989, doc. 21.

[86] Scamuzzi 1966 ; Williams \& Logan 1987, fig. 15. et la saisie de prisonniers n'est pas inconnue et rappelle le scellé de Den où le roi harponneur faisait face aux ennemis décapités (fig. 5). Dans les deux cas, l'artiste a voulu réunir la victoire sur les forces chaotiques du monde animal - les bêtes sauvages chassées - et du monde humain - les prisonniers [83]. Les deux céramiques proviennent de la même sépulture U-415, appartenant au cimetière de l'élite $U$, à Umm el-Qaab, Abydos. Or, ce cimetière concentre une grande quantité de matériel artistique prenant la forme d'un hippopotame : vases White Cross-lined, figurines en argile, et même une défense d'hippopotame [84]. Dès les origines, la mainmise sur cette force de la nature est gage de l'autorité et de la domination des classes supérieures. Ajoutons que quelques siècles plus tard, Abydos, berceau de l'élite naissante, devient la nécropole des premiers rois, et la mise à mort de I'hippopotame par le roi Den apparaît comme une activité héroïque héritière d'une longue tradition locale.

La chasse à I'hippopotame ne décore pas uniquement les vases White Cross-lined au Prédynastique; on la retrouve également incisée sur une palette rhomboïdale [85] ou peinte sur le tissu en lin de Gebelein [86]. Sur ce dernier, elle est accompagnée d'une procession de bateaux - dont le plus imposant pourrait transporter à son bord un prisonnier ligoté [87] - et d'un groupe de danseurs et danseuses. L'ensemble des décorations du tissu a été lu par B. Williams et T. Logan comme les différentes étapes rituelles du cycle jubilaire [88].

Un dernier type de médium, utilisé à profusion par les Nagadiens, porte les gravures d'hippopotames pris au piège : les parois rocheuses qui bordent le Nil et les ouadis des déserts oriental et occidental [89]. Là encore, les parties de chasse se jouent parmi d'autres décors : bateaux, parfois halés, animaux du désert, crocodiles... Une gravure du Ouadi Gash [90] présente notamment une scène de harponnage de deux pachydermes;
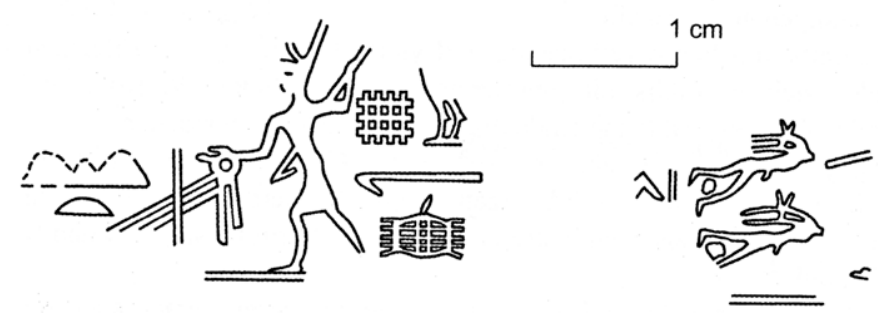

Figure 5 : scellé au nom du roi Den. D’après MülLer 2008, Fig. 3.

[87] Adams \& Ciałowicz, 1997, p. 36.

[88] Williams \& Logan 1987, p. 255-256.

[89] Cf. par exemple WinkLER 1938, pl. XIV, 2 ; DARNeLL 2009, fig. 8.

[90] WinKLER 1938, pl. XIV, 2, site 18 M 141a ; HENDRICKX et al. 2009, p. 173 , fig. 4. 
un peu en contrebas des chasseurs, un homme, un bâton en main, porte une coiffure un peu particulière : la couronne rouge, cette même couronne que porte le roi Den lorsqu'il part chasser I'hippopotame.

Mais cette chasse à I'hippopotame, si répandue dans I'iconographie nagadienne, a-t-elle une réalité historique? Assurément, I'hippopotame était chassé au Prédynastique ; c'est même l'espèce sauvage la plus couramment chassée dans le Nord du pays [91]. À l'inverse les restes fauniques de l'animal sont peu nombreux sur les sites de HauteÉgypte [92]. Cette différenciation s'explique sans doute par des biotopes distincts, le pachyderme ayant probablement de meilleures conditions de vie en Basse-Égypte [93]. V. Linseele et W. Van Neer proposent d'expliquer I'intense activité cynégétique sur les sites septentrionaux par le besoin de protéger les champs de l'activité destructrice de I'hippopotame [94]. Rappelons que quelques siècles plus tard une relation étroite existait entre la mise à mort ritualisée de I'hippopotame et la Basse-Égypte, attestée par la couronne rouge qui coiffe systématiquement la tête du roi dans les représentations. La profusion des restes fauniques dans le Delta au Prédynastique pourrait donc aussi être un indice témoignant de l'origine préhistorique de cette pratique rituelle.

La dimension rituelle de la traque de I'hippopotame est également visible en Haute-Égypte. Ici, le décalage entre la surexposition de cette activité dans la production artistique et les restes peu nombreux dans le matériel archéologique révèle le statut particulier de la chasse des espèces sauvages, probablement réservée à l'élite [95]. Le phénomène est illustré dans le complexe cultuel HK29A de Hiérakonpolis, qui conserve des fosses de déchets encerclant une cour. Ces dépotoirs étaient remplis d'ossements de diverses espèces sauvages, dont I'hippopotame. Les animaux ont probablement été consommés lors de festivités réservées aux membres de l'élite, en lien avec des activités rituelles [96]. Toujours à Hiérakonpolis ont été découverts trois hippopotames entiers dans le cimetière de I'élite HK6 [97]. Les animaux étaient enterrés en association avec des défunts et séparément, dans leurs propres tombes. Il est remarquable que I'hippopotame inhumé dans l'emplacement nommé Feature $H$, ainsi que d'autres espèces sauvages provenant de la nécropole HK6, présentent une fracture cicatrisée.

[91] Linseele \& Van Neer 2010, p. 57, tab. 2 et 3 ; Lesur 2013, p. 46. [92] Linseele \& Van Neer 2010, p. 57, tab. 2 et 3.

[93] Ibid.

[94] Ibid., p. 56 et 57. Le pachyderme pourrait également avoir été chassé pour son ivoire, utilisé dès le Prédynastique dans l'artisanat : KRZYSZKOWSKA \& MORKOT 2000, p. 326-327.

[95] HENDRICKX 2010.
Située sur la partie inférieure de son péroné, elle permet d'envisager le scénario suivant : attaché grâce à une corde qui enserre sa jambe, l'animal s'est brisé I'os en tentant de s'enfuir, sans succès [98]. Le jeune hippopotame a ensuite été maintenu en captivité pendant une période assez longue pour que sa fracture puisse cicatriser [99], avant d'être enterré avec quelque personnage de haut rang.

Des indices tendent par ailleurs à montrer que le pachyderme pouvait déjà posséder les deux aspects positif et négatif qui le caractériseront plus tard. Le côté positif, protecteur et régénérant de l'animal est visible sur les céramiques White Cross-lined. Ici, les hippopotames, quand ils ne sont pas chassés, sont très souvent associés aux motifs végétaux, synonymes de fertilité [100]. La structuration des décors du vase D1187 du musée d'Art et d'Histoire de Genève montre la position interchangeable des hippopotames et des végétaux (fig. 9). La réunion des motifs végétaux avec la figure de I'hippopotame rappelle les figurines de I'animal en faïence, déposées dans les tombes du Moyen Empire et ornées de décors végétaux et aquatiques :

Figure 9 : vase White Cross-lined, provenance inconnue. D'après WILD 1948, Fig. 2.
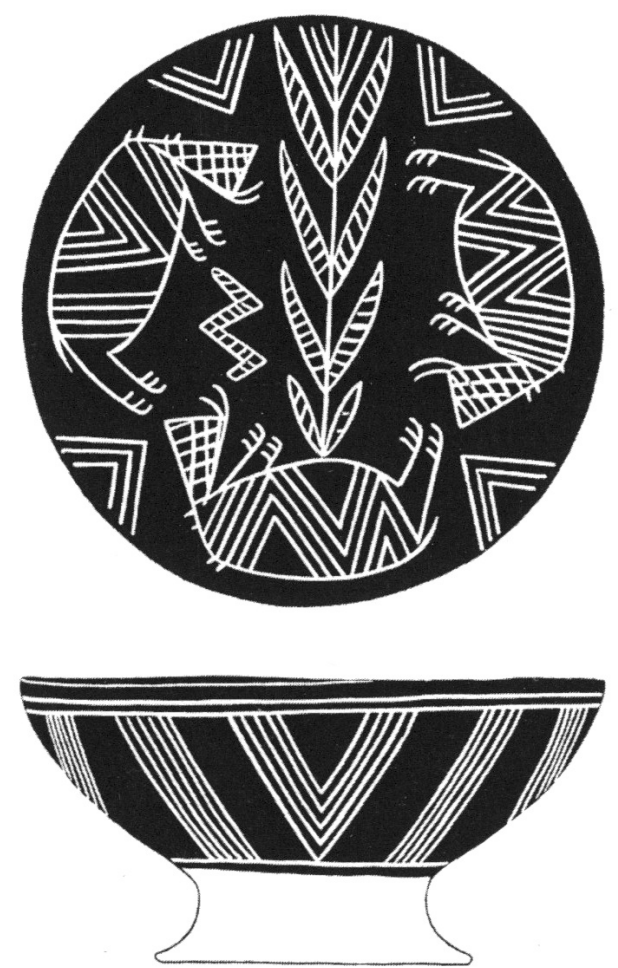

[96] Linseele, Van Neer \& Friedman 2009 ; Linseele \& Van Neer 2010, p. 56 ; HENDRICKX 2010, p. 125-127.

[97] Découverts dans les tombes 2, 12 et Feature $H$ : VAN NeER \& LINSEELE 2009, p. 12.

[98] Van Neer \& Linseele 2009, p. 12.

[99] Ibid.

[100] GrafF 2009, n40, 55, 70, 73, 97, 98, 106, 127, 134, 164. 
le décor exacerbe leur fonction régénérative [101]. Le lien entre les pachydermes et la fertilité paraît encore plus explicite sur I'un des vases de la tombe U-415, dont les décorations étaient interprétées plus haut comme une simple chasse à l'hippopotame associée à la prise de captifs (fig. 7). Or, dans ce contexte, un détail surprend : deux des hippopotames chassés sont gravides, leurs petits soigneusement représentés dans les ventres de leurs mères. Une observation attentive de la seconde céramique découverte dans la tombe permet d'y déceler un détail analogue : un des personnages prisonniers possède un ventre rebondi, qui pourrait évoquer celui d'une femme enceinte [102] (fig. 8). Ce détail pourrait indiquer un rapprochement entre les hippopotames et la figure de la femme enceinte, qui véhiculeraient tous deux le champ sémantique de la fertilité. Pourrait-on voir dans ces indices de grossesse un signe annonciateur de la future déesse hippopotame Thouéris, protectrice des femmes enceintes ?

Il faut par ailleurs insister sur le fait que ces animaux ne sont pas représentés tués, mais bien en cours de capture [103], de même pour les prisonniers. Ceci pourrait dénoter la volonté de s'emparer de leur pouvoir régénératif en les capturant. Ce détail est à mettre en lien avec les hippopotames enterrés dans le cimetière de l'élite à Hiéraconpolis. Tous très jeunes - un des trois est même nouveau-né -, ils ont peut-être été retenus en captivité pour être ensuite enterrés avec les membres de I'élite afin de faciliter leur régénération en prenant part à quelque rite de renaissance [104].

Toutefois, la présence de ces hippopotames dans les tombes de personnages de haut rang pourrait aussi s'expliquer par le caractère négatif de I'hippopotame. En effet, les animaux pourraient correspondre à des trophées de chasse, que l'élite aurait voulu emporter dans l'au-delà pour manifester sa suprématie sur les forces destructrices de la nature. C'est probablement pour servir le même besoin de domination qu'elle a fait représenter la chasse de I'hippopotame sur quantité d'objets. La présence dans une tombe du cimetière HK6 d'une figurine d'hippopotame brisée et de couleur rouge, synonyme de chaos, doit probablement renvoyer elle aussi à l'aspect négatif du colosse aquatique.

Dès le Prédynastique, I'hippopotame pourrait ainsi véhiculer une image ambivalente, à la fois positive et négative. Sa chasse tient une place prépondérante dans la vie des Nagadiens : elle est représentée à maintes reprises

[101] VeRnUS 1991, p. 337-338.

[102] Ce détail fait pendant à un vase découvert dans la tombe U239 d'Abydos sur lequel est également dépeint un individu au ventre proéminent. Cf. DREYER et al. 1998, fig. 13.

[103] HENDRICKX \& EYCKERMAN 2012, p. 58-59.

[104] Linseele \& VAN NeER 2010, p. 64. dans la production artistique, et sa réalité historique est démontrée par les sources archéozoologiques. Le croisement de ces données a permis de comprendre que la traque de cet animal aquatique était en lien étroit avec les personnages de haut rang et pouvait posséder une dimension rituelle. Ainsi, la mise à mort rituelle de I'hippopotame par le roi Den est sans doute héritière d'une tradition préhistorique, tradition qui se perpétue durant toute la période pharaonique.

\section{... ET QUI SE PERPÉTUE Ä L'ÉPOQUE PHARAONIQUE}

De fait, la chasse aux hippopotames, loin d'être abandonnée au début de la période historique, se perpétue, au moins par la vertu des media iconographique et épigraphique, durant toute la période pharaonique jusqu'à la Basse Époque. Elle est ainsi abondamment représentée sur les parois des tombes de particuliers de I'Ancien au Nouvel Empire. Au cours de I'Ancien Empire et de la Première Période intermédiaire, le maître ne fait qu'assister à la scène du harponnage, effectuée par

Figure 10 : scène de chasse à l'hippopotame, tombe de Ti, Saqqara, Ancien Empire. D'après WILD 1966, pl. 115.

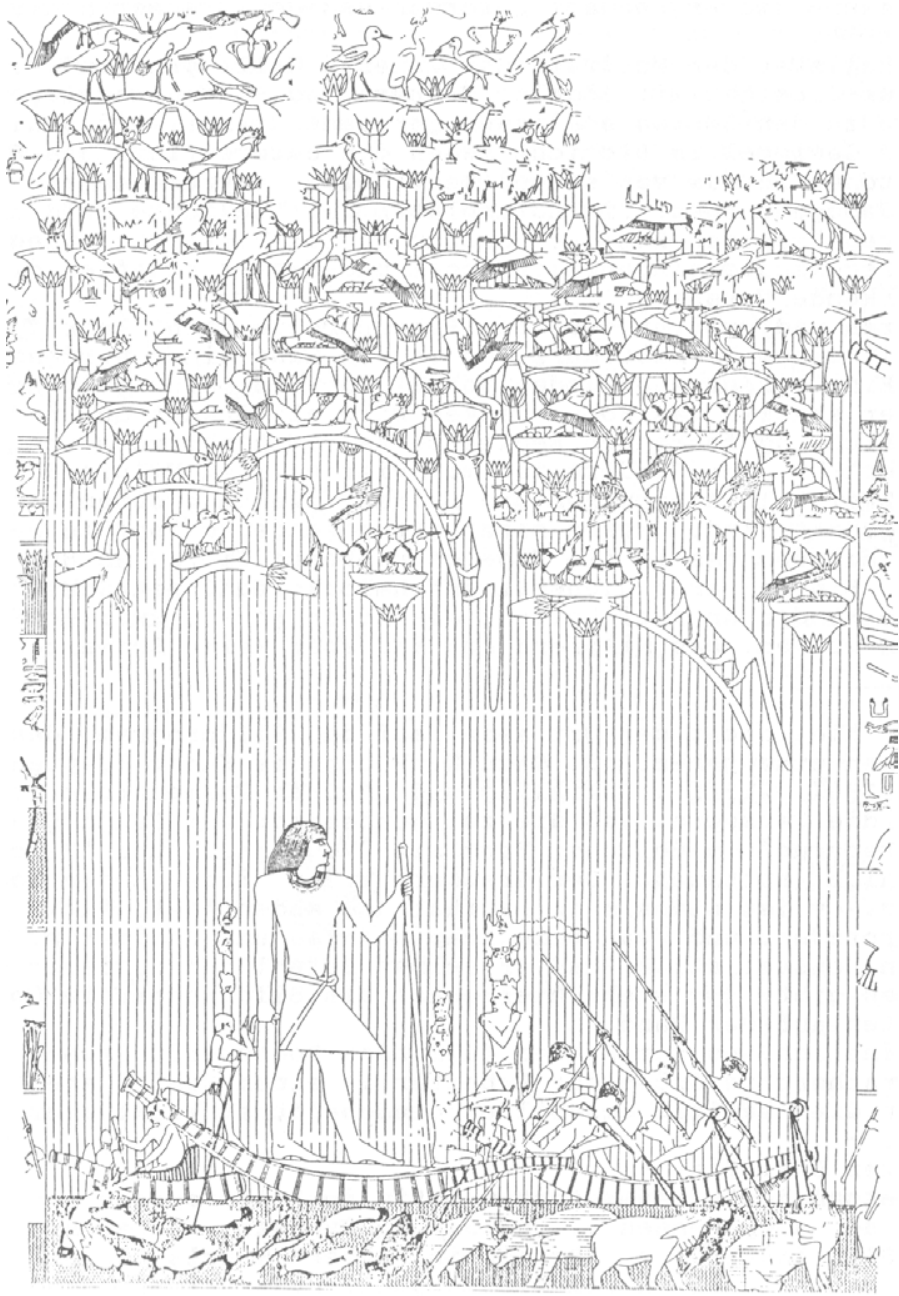




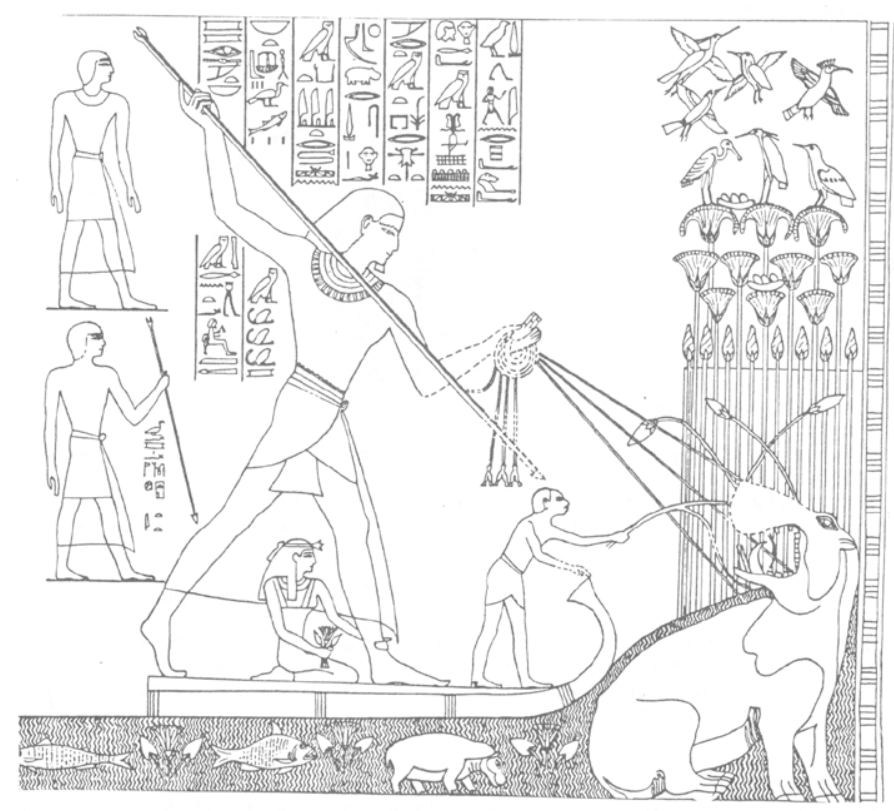

Figure 11 : scène de chasse à l'hippopotame, tombe d'Antef, TT 155, Thèbes, XVIII e dynastie. D'après SÄVE-SöDERBERGH 1953, Fig. 1.

plusieurs hommes, sans doute dans le but de protéger son domaine agricole [105] (fig. 10); dans les tombes du Nouvel Empire en revanche, le défunt harponne luimême et seul la bête (fig. 11) [106]. Si aucun noble ne s'occupe personnellement de la chasse dans la documentation de l'Ancien Empire, c'est peut-être que sa mise à mort -exceptée celle indispensable, exécutée par les paysans pour prévenir les dommages aux cultures - était à cette époque un privilège royal [107]. En effet, introduite par Den à la $1^{\text {re }}$ dynastie, la chasse ritualisée de I'hippopotame est reproduite par la figure royale durant toute la période pharaonique [108]. Le plus ancien témoignage royal, malheureusement très fragmentaire, proviendrait du temple funéraire de Sahourê, deuxième roi de la Ve dynastie [109]. La chasse royale est en revanche incontestable sur une scène incomplète mais reconstituée, découverte dans un temple funéraire érigé en I'honneur de Pépi II, sous la VIe dynastie à Saqqara [110] (fig. 12). On y retrouve les techniques de chasse déjà éprouvées durant les périodes précédentes. Des images comparables du roi harponneur sont figurées sur deux documents datés du règne d'Amenemhat III (XII dynastie) :

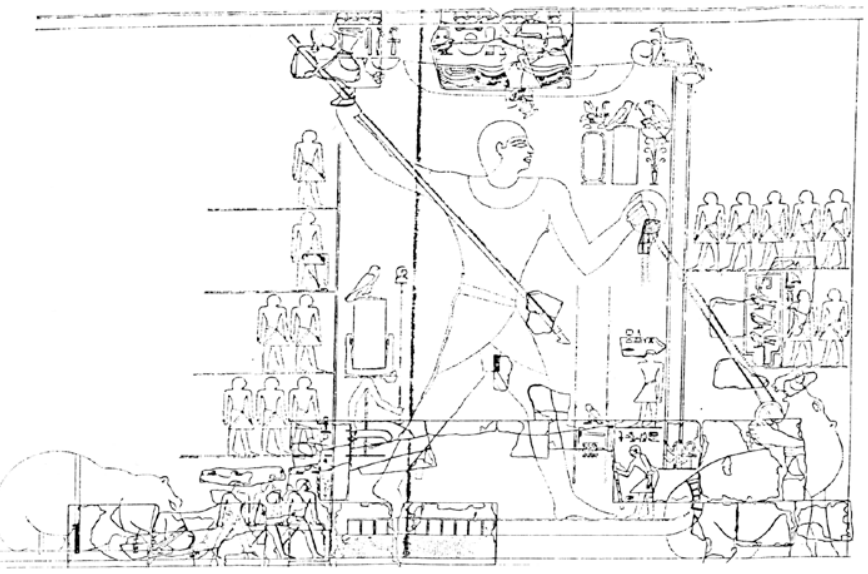

Figure 12 : scène de chasse royale à l'hippopotame, temple funéraire de Pépi II, Saqqara, Vle dynastie. D’après JÉQUIER 1940, pl. 32.
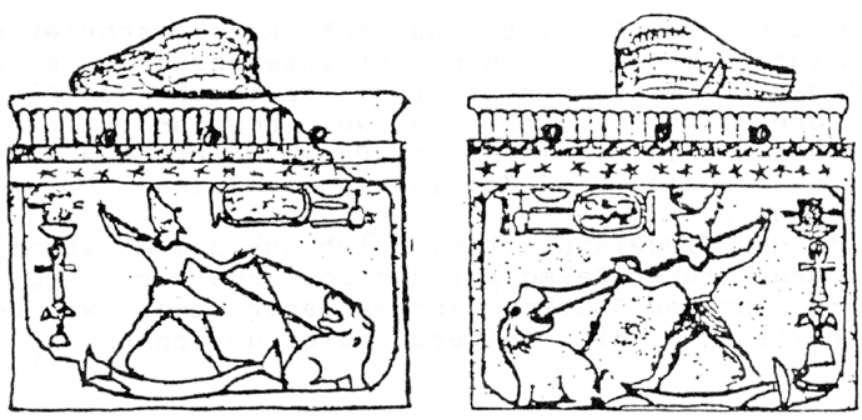

Figure 13 : porte-amulette d'Amenemhat III, Tell Basta, XIle dynastie. D'après FARID 1964, Fig. 6.

un scarabée [111] et un porte-amulette [112]. Ce dernier comporte une scène de harponnage sur les deux faces : coiffé de la blanche sur la première face, le monarque arbore la couronne de rouge sur la seconde (fig. 13). C'est aussi la couronne de Basse-Égypte qui coiffe la tête du souverain harponneur incisé sur un scarabée privé de la Seconde Période intermédiaire [113] (fig. 14). Le Nouvel Empire est également riche d'images du monarque en chasseur d'hippopotame: on citera un scarabée au nom de Thoutmosis III [114], deux statues d'Amenhotep II coiffé de la couronne rouge [115], une statue de Thoutmosis IV [116], deux autres attribuées à Toutânkhamon [117], deux peintures murales de Séthy II portant la couronne blanche [118] (fig. 15) et une
[105] VAndier 1964, p. 775 ; BeHRMAnn 1989, doc. 74 à 98 ; YOYOTte 2005, p. 255.

[106] VANDiER 1964, p. 775 ; Behrmann 1989, doc. 161 à 174. Cette différence repose selon T. Säve-Söderbergh sur le fait que les scènes du Nouvel Empire ne découleraient pas des images provenant des tombeaux de particuliers de I'Ancien Empire, mais des prototypes royaux (SÄve-SöderberGH 1953, p. 15-16).

[107] Le privilège de se faire représenter en train de chasser I'hippopotame aurait ensuite été accordé de manière exceptionnelle à quelques hauts dignitaires au début du Nouvel Empire (YoYOTtE 2005, p. 255). Cf. également note précédente.

[108] Säve-SÖDERBERGH 1953.
[109] BorCHARDT 1913, p. 29-30, pl. 16 ; SäVE-SÖDERBERGH 1953, p. 19. [110] JÉQuier 1940, pl. 32 ; BeHRMAnn 1989, doc. 94.

[111] New York, Metropolitan Museum, 26.2.1.

[112] Behrmann 1989, doc. 133a.

[113] LACOVARA 1992, p. 21, fig. 6b.

[114] BeHRMAnN 1989, doc. 160.

[115] Ibid., doc. 167a.

[116] Ibid., doc. 167b.

[117] Ibid., doc. 167c.

[118] Ibid., doc. 167d. 


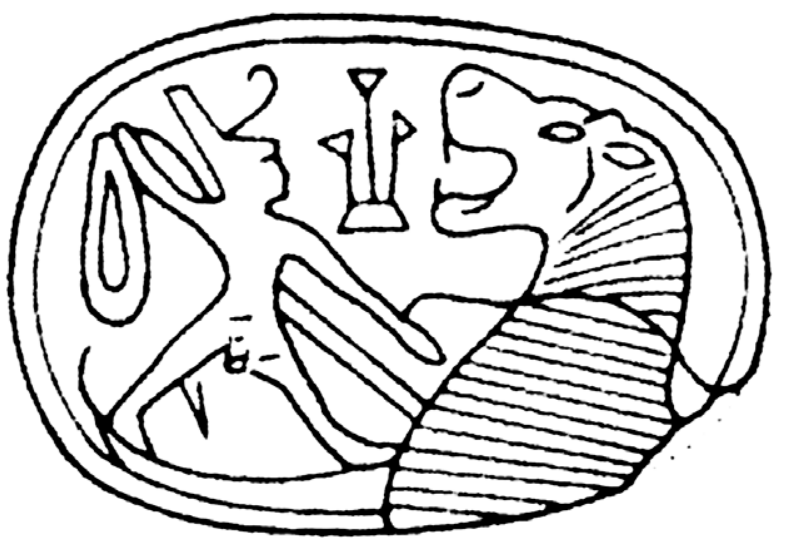

Figure 14 : scène de chasse royale à l'hippopotame sur un scarabée en stéatite, Deuxième Période intermédiaire. D'après LACOVARA 1992, Fig. 6b, dessin de Y. Markowitz.

paroi dépeignant Taousert [119]. La récurrence de cette image du roi harponneur révèle l'importance de ce thème, véritable canon artistique au même titre que la figure du roi massacrant les ennemis.

Des souverains chasseurs d'hippopotames : la nature des exécuteurs aurait pu s'arrêter à ce haut degré. Pourtant des épisodes mythiques placent des divinités dans le rôle du chasseur d'hippopotame. La première mention d'un dieu harponneur survient dans les Textes des pyramides [120], et prend la forme de Hémen, dieu-faucon maître de Héfat. L'implication de cette divinité dans la mise à mort d'un hippopotame est confirmée par une représentation du tombeau d'Ânkhtyfy, à Mo'alla [121]. Elle figure un défilé nautique accompagné d'une chasse à l'hippopotame réalisée par les fils du prince et dirigée par ce dernier [122]. La légende précise que la célébration est « I'affaire de Hémen » [123].

Si la première attestation d'un dieu combattant I'hippopotame est Hémen, l'adversaire divin par excellence du colosse aquatique demeure Horus. Le combat est narré dans le mythe d'Horus et Seth, où ce dernier prend la forme du dangereux pachyderme au cours d'un épisode. Il existe plusieurs versions de la querelle, relatée dans les calendriers des jours fastes et néfastes [124], dans les Aventures d'Horus et Seth [125] ou encore sur les parois du temple ptolémaïque d'Edfou [126]. Selon les variantes, soit seul Seth se transforme en hippopotame pendant qu'Horus garde sa forme humaine, soit les deux frères se métamorphosent. Si le déroulement du combat varie, sa raison reste identique : obtenir sa place sur le trône pour gouverner le Double Pays. Dans ce mythe, I'hippopotame harponné représente Seth, mais il symbolise aussi plus généralement tous les ennemis du roi et du dieu qui doivent être anéantis
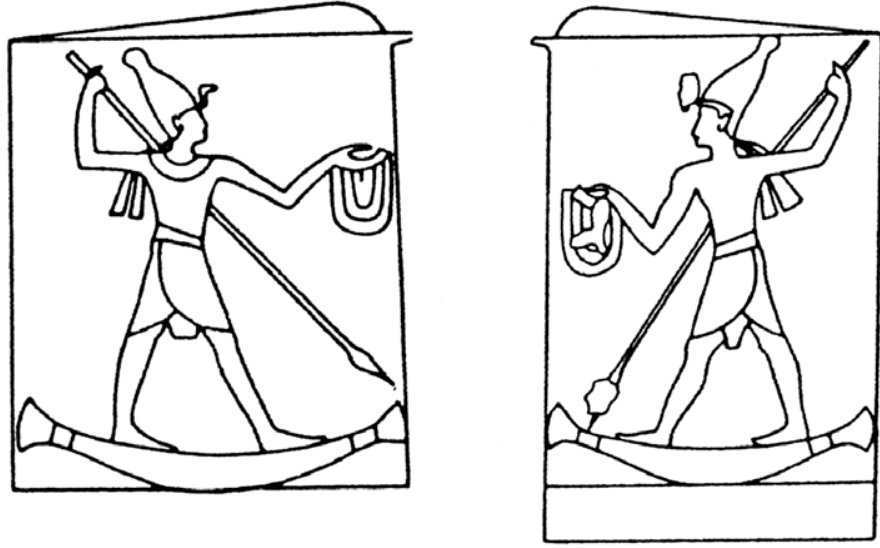

Figure 15 : scène de harponnage sur une paroi murale, tombe de Séthy II, KV 15, Thèbes, $\mathrm{XIX}^{\mathrm{e}}$ dynastie. D'après BEHRMANN 1989, doc. 167d

avant le couronnement [127]. Parfois, c'est le roi qui prend la place d'Horus et harponne I'hippopotame, sous les yeux de ce dernier. Ainsi, quand ce n'est pas Horus qui tue I'hippopotame pour le bénéfice du roi, c'est le roi qui tue I'animal en I'honneur d'Horus [128]. Cette position interchangeable du roi et d'Horus est tout à fait cohérente : Horus n'est-il pas le prototype du roi régnant?

Si à partir de I'Ancien Empire la chasse à I'hippopotame était semble-t-il autorisée pour protéger les cultures des ravages, la mise à mort rituelle du pachyderme était une affaire réservée, sauf exception, aux dieux et au souverain [129]. Son exécution, au moins par la vertu de l'image et des écrits, permettait au roi de montrer sa domination sur les forces destructrices de la nature incarnées par l'hippopotame et de s'approprier leurs pouvoirs, tout en accomplissant une des fonctions primordiales de Pharaon : maintenir l'ordre cosmique [130].

[119] Ibid., doc. 167e.

[120] Pyr. 235a-b.

[121] Première Période intermédiaire. VANDIER 1946; SÄVe-SöderberGH 1953, p. 29-33 ; VandieR 1964, p. 781-783

[122] VANDIER 1946, p. 94.

[123] YoYotTe 2005, p. 254.

[124] Papyrus du Caire no 86637 : BAKIR 1966, pl. VII 4-VIII 3 ; Behrmann 1989, doc. 185b.1; Papyrus Sallier IV 2,6-3,5 : BeHRMAnN 1989, doc. 185b.2.

[125] BROZE 1996, p. 74-79.

[126] Behrmann 1989, doc. 228. Sur le harponnage de I'hippopotame dans le temple d'Edfou, cf. LABRIQUe 1993.

[127] Säve-SöDERBERGH 1953, p. 29.

[128] Ibid.

[129] Cf. note 106.

[130] BonhêMe \& Forgeau 1988, p. 132-139. 


\section{CONCLUSION}

Avec comme point de départ une entrée de la pierre de Palerme, dont la lecture «transpercer I'hippopotame » a été vérifiée, cette enquête, en compilant les sources contemporaines qui relateraient un événement similaire, a permis de révéler l'importance de la chasse ritualisée de I'hippopotame durant le règne de Den.

Une incursion dans la documentation prédynastique a révélé par ailleurs l'ancienneté de cette pratique cynégétique. Dès cette époque reculée, des indices iconographiques et archéologiques révèlent que I'hippopotame présentait déjà dans la pensée nagadienne deux aspects, I'un positif et protecteur, l'autre négatif et destructeur. Abondamment représentée, la chasse au pachyderme aquatique devait dès lors véhiculer un message fort de puissance et être considérée comme un acte héroïque. Sa surreprésentation dans l'iconographie, associée à la présence de I'animal à la fois sur le site cultuel HK29A et dans la nécropole de l'élite à Hiérakonpolis, révèle le lien étroit entre la chasse à I'hippopotame et les classes dirigeantes dès le Prédynastique. La traque de ce colosse du Nil dénote la volonté de l'élite d'affirmer son pouvoir en dominant les forces destructrices de la nature.

Avec l'intronisation des premiers pharaons à la période thinite, la mise à mort de l'hippopotame semble évoluer d'une activité élitaire à un privilège exclusivement royal. Son rôle est si important dans la vie du souverain qu'elle est consignée dans les annales. Comme la plupart des entrées de la pierre de Palerme, le harponnage du pachyderme relève d'une activité rituelle [131]. Héritière d'une longue tradition prédynastique, cette pratique se déroulait probablement au cours d'un festival. En effet, des fêtes royales [132] de plusieurs sortes sont déjà attestées à cette époque reculée, dont la célèbre fêtesed. On connaît par ailleurs l'existence, dès le début de I'Ancien Empire, d'une cérémonie royale destinée à « la Blanche », déesse-hippopotame [133]. L'hypothèse d'un festival durant le règne de Den consacré à la chasse ritualisée du pachyderme est du reste renforcée par le nombre de sources contemporaines du souverain figurant cet épisode.

La mise à mort du colosse aquatique se perpétue ensuite durant toute la période pharaonique, témoignant de l'im-

[131] Cf. WiLKINSON 2000, p. 66-71.

[132] WiLKINSON 1999, p. 208-222.

[133] Cf. introduction. BeHRMANn 1989 : doc. 62, 63, 72, 159, 196. Cf. Säve-Söderbergh 1953, p. 45-55; KaISer 1988, 1997 ; AltenmülleR 1994 ; YOYOTTE 2005, p. 259-260.

[134] Manetho, Aegyptiaca, Fr. 6 (de Sextus Julius Africanus). S'il est tout à fait possible que la mort de Ménès due à un hippopotame, relatée par Manéthon, puise dans une réalité historique d'un combat entre un des rois fondateurs et un portance de cette pratique rituelle pour la monarchie royale. Toutefois, une question demeure : la mise à mort rituelle de l'hippopotame par le roi procède-t-elle d'une réalité historique ou de la pure fiction ? D'emblée on serait tenté d'opter pour le simulacre. On connaît en effet la légendaire férocité de l'animal lorsqu'il est attaqué ; il est également indéniable que les représentations du roi harponnant seul I'hippopotame, voire le combattant à mains nues, ne peuvent rendre la réalité. Toutefois cette pratique était-elle véritablement invraisemblable ? À en croire Manéthon, Ménès, pharaon fondateur et unificateur de l'Égypte, serait mort précisément sous les attaques d'un hippopotame [134]. N'est-il donc pas possible que les pharaons - à tout le moins leurs premiers représentants - aient combattu I'hippopotame, ou porté le coup de grâce à la bête agonisante ? En fait, I'hypothèse, au vu du contexte particulier de la période thinite, mérite réflexion. En effet, la royauté, tout juste naissante, a encore besoin d'affirmer son pouvoir politique. C'est donc par la réalisation d'actes forts et dangereux, en somme héroïques, voire surhumains, que le souverain pourra légitimer sa place sur le trône. La chasse d'une bête féroce comme l'hippopotame en est un parfait exemple. Par la suite, le rituel pourra inclure des simulacres, comme l'atteste le « Festival de la victoire » décrit sur les parois du temple d'Edfou, où un gâteau en forme d'hippopotame, transpercé de dix harpons, se substitue à I'animal [135].

Finalement, si la question reste ouverte, qu'elle soit réelle ou fictive, la mise à mort de I'hippopotame par le roi joue un rôle tout aussi déterminant. Cette pratique rituelle va permettre au souverain de montrer son courage et sa force surhumaine et ainsi pérenniser son statut de maître du Double Pays. Le choix, dès le Prédynastique, de I'hippopotame comme représentant des forces chaotiques à combattre, au détriment d'autres bêtes sauvages féroces, n'est certainement pas un hasard. P. Vernus a montré que I'hippopotame était un émissaire de l'eau primordiale, chaos originel duquel émerge toute création. Cependant, I'hippopotame n'est pas n'importe quel émissaire : contrairement au crocodile qui représente l'agent d'anéantissement, I'hippopotame, lui, est agent de résurrection [136]. En le tuant, le roi s'approprie son pouvoir destructeur et accomplit

hippopotame, le récit comporte indéniablement une part mythologique, la réalité historique de Ménès n'étant pas avérée. Cf. Vernus 1991.

[135] SÄVe-Söderbergh 1953, p. 26-27. P. Posener-Kriéger a par ailleurs proposé qu'une figurine d'hippopotame en bois découverte dans le temple funéraire de Rêneferef ( $V^{\mathrm{e}}$ dynastie) était pareillement destinée à mettre en action le rite de la mise à mort de I'hippopotame, à moins que la statuette ne représente un symbole de renaissance : Posener-Kriéger 1991, p. 298.

[136] VERNUS 1991. 
un de ses rôles principaux en tant que Pharaon : maintenir la Maât [137]. Cette lutte du souverain contre les forces négatives incarnées par l'hippopotame trouve son parallèle structural dans le mythe fondateur d'Horus et Seth, où Horus, prototype du roi régnant, combattra Seth, expression du désordre prenant les traits du pachyderme, pour gagner sa place sur le trône et gouverner l'Égypte. De fait, héritière d'une longue tradition préhistorique, puis partie constitutive d'un mythe fondateur, la chasse de I'hippopotame est une pratique rituelle essentielle dans le processus de légitimation de Pharaon. Devenue une figure stéréotypée de la production artistique pharaonique, l'image du roi harponneur participe ainsi à la construction de l'idéologie pharaonique.

[137] BONHÊME \& ForgeAU 1988, p. 132-139.

\section{BIBLIOGRAPHIE}

Adams, B. \& Ciakowicz, K., 1997, Protodynastic Egypt, Princes Risborough.

Allen, J.P., 2013, A New Concordance of the Pyramid Texts [en ligne], Brown University. Disponible à l'adresse https://www.dropbox.com/sh/0xo88uy04urnz0v/016_ojF8f_(consultation 2 avril 2014).

Altenmüller, H., 1994, «Das "Fest des Weissen Nilpferds" und das "Opfergefilde" », dans C. Berger, G. Clerc \& N. Grimal (éd.), Hommages à Jean Leclant. Vol. I, Études pharaoniques (BdE 106, 1), Cairo, p. 29-44.

Bakir, A., 1966, The Cairo Calendar no. 86637, Cairo.

Behrmann, A., 1989, Das Nilpferd in der Vorstellungswelt der Alten Ägypter. Teil I, Katalog, Frankfurt am Main - Berlin - Paris.

Bonhême, M-A. \& Forgeau, A., 1988, Pharaon : les secrets du pouvoir, Paris.

BorchardT, L., 1913, Das Grabdenkmal des Königs Sahu-Re, Leipzig.

Breasted, J.H., 1906, Ancient Records of Egypt. Vol. I. The First to the Seventeenth Dynasties, Chicago.

Broze, M., 1996, Mythe et roman en Égypte ancienne : les aventures d'Horus et Seth dans le papyrus Chester Beatty I, Leuven.

Champollion, J.-F., 1844, Monuments de l'Égypte et de la Nubie : notices descriptives conformes aux manuscrits autographes rédigés sur les lieux, Paris.

Czichon, R.M. \& Sievertsen, U., 1993, « Aspects of space and composition in the relief representations of the Gebel el-Arak knifehandle », Archéo-Nil 3, p. 49-55.

Darnell, J.C., 2009, « Iconographic Attraction, Iconographic Syntax, and Tableaux of Royal Ritual Power in the Pre- and ProtoDynastic Rock Inscriptions of the Theban Western Desert », Archéo-Nil 19, p. 83-107.

DE Jong, A., 2001, s. v. Hippopotami, The Oxford Encyclopedia of Ancient Egypt, vol. II, p. 100-101.

DE WITT, C., 1958, « Une représentation rare au Musée du Cinquentenaire », Chronique d'Égypte XXXIII, 65, p. 24-28.

Dreyer, G., Hartung, U., Hikade, T., Köhler, E.C., Müller V. \& Pumpemmeier, F., 1998, « Umm el-Qaab. Nachuntersuchungen im frühzeitlichen Königsfriedhof. 9./10 Vorbericht », Mitteilungen des Deutschen Archäologischen Instituts, Abteilung Kairo 54, p. 77-167.

Dreyer, G., Hartmann, R., Hartung, U., Hikade, T., Köpp, H., Lacher, C., Müller, V., Nerlich, A. \& Zink, A., 2003, « Umm elQaab. Nachuntersunchungen im frühzeitlichen Königsfriedhof. 13./14./15. Vorbericht », Mitteilungen des Deutschen Archäologischen Instituts, Abteilung Kairo 59, p. 67-138.

Dreyer, G., Blöbaum, A.I., Engel, E.-M., KöPp, H., Müller, V., 2011, « Umm el-Qaab. Nachuntersunchungen im frühzeitlichen Königsfriedhof. 19./20./21. Vorbericht », Mitteilungen des Deutschen Archäologischen Instituts, Abteilung Kairo 67, p. 53-92.

Droux, X., 2011, « Twinned Hippopotamus Figurines of the Predynastic Period », dans R.F. Friedman \& P.N. Fiske (éd.), Egypt at its origins 3. Proceedings of the International Conference "Origin of the State. Predynastic and Early Dynastic Egypt", London, 27th July - 1st August 2008 (OLA 205), Leuven, p. 349-377.

FARID, S., 1964, « Preliminary Report on the Excavations of the Antiquities Department at Tell Basta (Season 1961) », Annales du Service des Antiquités de l'Égypte 58, p. 85-98.

Godron, G., 1990, Études sur l'Horus Den et quelques problèmes de l'Égypte archaïque, Genève.

GrafF, G., 2009, Les peintures sur vases de Nagada I - Nagada II : nouvelle approche sémiologique de l'iconographie prédynastique, Leuven.

GundLACH, R., 1986, s. v. Thoeris, Lexikon der Ägyptologie, vol. VI, col. 494-497.

HaRtung, U., 2010, « Hippopotamus hunting and bureaucrats Elite burials at cemetery U at Abydos », dans F. Raffaele, M. Nuzzolo \& I. Incordino (éd.), Recent discoveries and latest researches in Egyptology. Proceedings of the First Neapolitan Congress of Egyptology, Naples, June 18th-20th 2008, Wiesbaden, p. 107-119.

Hendrickx, S., 1998, «Peaux d'animaux comme symboles prédynastiques », Chronique d'Égypte 73, p. $103-130$.

Hendrickx, S., 2006, «Predynastic - Early Dynastic Chronology », dans E. Hornung, R. Krauss \& D. Warburton (éd.), Ancient Egyptian Chronology, Leiden - Boston, p. 74-77. 
Hendrickx, S., 2010, «L'iconographie de la chasse dans le contexte social prédynastique », Archéo-Nil 20, p. 106-133.

Hendrickx, S. \& Depraetere, D., 2004, « A theriomorphic Predynastic stone jar and hippopotamus symbolism », dans S. Hendrickx, R.F. Friedman, K.M. Ciałowicz \& M. Chłodnicki (éd.), Egypt at its origins. Studies in memory of Barbara Adams. Proceedings of the international conference "Origin of the State. Predynastic and Early Dynastic Egypt", Kraków, 28th August - 1st September 2002 (OLA 138), Leuven, p. 801-822.

Hendrickx, S., Swelim, N., Raffaele, F., Eyckerman, M. \& Friedman, R.F., 2009, « A lost Late Predynastic-Early Dynastic royal scene from Gharb Aswan », Archéo-Nil 19, p. 169-178.

Hendrickx, S. \& Eyckerman, M., 2012, « Visual representation and state development in Egypt », Archéo-Nil 22, p. $23-72$.

Houser-Wegner, J., 2001, s. v. Taweret, The Oxford Encyclopedia of Ancient Egypt, vol. III, p. 100-101.

JÉQUier, G., 1940, Fouilles à Saqqarah. Le Monument funéraire de Pepi II. Tome III, les approches du temple, Cairo.

JUNKER, H., 1940, Gîza IV. Die Mastaba des Kai-em-anch, Wien - Leipzig.

KAISER, W., 1988, «Zum Hb-Hd.t.t », Mitteilungen des Deutschen Archäologischen Instituts, Abteilung Kairo 44, p. 125-134.

KAISER, W., 1997, « Noch einmal zum H. b-H. d.t. », Mitteilungen des Deutschen Archäologischen Instituts, Abteilung Kairo 53, p. $113-115$.

KAPLONY, P., 1963, Die Inschriften der ägyptischen Frühzeit, Wiesbaden.

Krzyszkowska, O. \& Мовкот, R., 2000, « Ivory and related materials », dans P. T. Nicholson \& I. Shaw (éd.), Ancient Egyptian Materials and Technology, Cambridge, p. 320-331.

LABRIQUE, F., 1993, «"Transpercer l'âne" à Edfou », dans J. Quaegebeur (éd.), Ritual and sacrifice in the ancient Near East, Proceedings of the International conference from the 17th to the 20th of April 1991, Leuven, p. 175-189.

Lacovara, P., 1992, «A New Date for an Old Hippopotamus », Journal of the Museum of Fine Arts 4, p. 17-26.

LÉon L'Africain, 1993, Description de l'Afrique. Volume III, Islamic geographic, vol. 138, Wolfgang Goethe University, Frankfurt

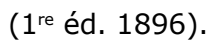

LePSIUS, R., 1842, Auswahl der wichtigsten Urkunden des aegyptischen Alterthums, Leipzig.

Lesur, J., 2013, «Des animaux et des hommes en Égypte au Néolithique et Prédynastique. Les apports de l'archéozoologie », Archéo-Nil 23, p. 33-54.

Linseele, V., Van Neer, W. \& Friedman, R.F., 2009, « Special animals from a special place? The fauna from HK29A at Predynastic Hierakonpolis », Journal of the American Research Center in Egypt 45, p. 105-136.

Linseele, V. \& VAN Neer, W., 2010, « Exploitation of desert and other wild game in ancient Egypt : The Archaeozoological evidence from the Nile Valley », dans H. Riemer, F. Förster, M. Herb \& N. Pöllath (éd.), Desert animals in the eastern sahara. Status, economy, significiance, and cultural reflection in antiquity, Proceedings of an interdisciplinary ACACIA Workshop held at the University of Cologne, December 14-15 2007, Köln, p. 47-78.

Manetho. Aegyptiaca, éd. W.G. Waddell, 1948, Cambridge - London.

Manlius, N., 2000, «Biogéographie et Écologie historique de l'hippopotame en Égypte », Belgian Journal of Zoology $130, \mathrm{n}^{\circ} 1$, p. 59-66.

MüLleR, V., 2008, « Nilpferdjagd und Geköpfte Feinde - Zu Zwei Ikonen des Feindvernichtungsrituals », dans E. Engel, V. Müller \& U. Hartung (éd.), Zeichen aus dem Sand. Streiflichter aus Ägyptens Geschichte zu Ehren von Günter Dreyer, Wiesbaden, p. 477-493.

MüLleR, V., 2014, « Seal impressions from Den's tomb at Abydos: New evidence and new interpretations », communication présentée à Origins 5. Fifth international conference of Predynastic and Early Dynastic Studies, Cairo.

NewberRY, P.E., 1893, Beni Hasan. Part I, London.

NewberRy, P.E., Wainwright, G.A., 1914, « King Udy-Mu (Den) and the Palermo Stone », Ancient Egypt, p. 148-155.

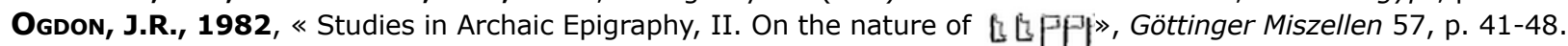

Osborn, D.J. \& OsbornovÁ, J., 1998, The Mammals of Ancient Egypt, Warminster.

Payne, C.J., 1993, Catalogue of the Predynastic Egyptian Collection in the Ashmolean Museum, Oxford - New York.

Paton, D., 1915, Early Egyptian records of travel. I. To the end of the XVIIth dynasty, Princeton - London - Oxford.

Petrie, W.M.FI., 1900a, Dendereh 1898, London.

Petrie, W.M.FI., 1900b, The Royal Tombs of the First Dynasty, Part I, London.

Petrie, W.M.FI., 1901, The Royal Tombs of the First Dynasty, Part II, London.

Petrie, W.M.FI., 1902, Abydos I, London.

Pirez, A.V., 2012, « Hippo goddesses of the Egyptian Pantheon », dans H.A. El Gawad, N. Andrews, M. Correas-Amador, V. Tamorri \& J. Taylor (éd.), Current Research in Egyptology 2011, Proceedings of the Twelfth Annual Symposium, Oxford, p. $210-220$.

Posener-KriéGer, P., 1991, «Quelques pièces du matériel cultuel du temple funéraire de Rêneferef », Mitteilungen des Deutschen Archäologischen Instituts, Abteilung Kairo 47, p. 293-304.

QuibelL, J. \& Green, F., 1902, Hierakonpolis, Part II, London.

Säve-SöderberGH, T., 1953, On Egyptian representations of hippopotamus hunting as a religious motive, Uppsala.

Scamuzzi, E., 1966, L'art égyptien au musée de Turin, Paris.

SCHÄFER, H., 1902, Ein Bruchstück altägyptischer Annalen, Berlin.

Seligman, C.G., 1916, « An Early Representation of Taurt », Ancient Egypt, Part II, p. 53.

Van Neer, W. \& Linseele, V., 2009, « Animal Hospital: Healed Animal Bones from HK6 », Nekhen News 21, p. 11-12.

VANDier, J., 1946, « Hémen, maître de Héfat, et I'hippopotame », Revue de l'histoire des religions 132, p. 93-97.

VANDIER, J., 1952, Manuel d'archéologie égyptienne. Tome I, Les époques de formation, Paris.

VANDIER, J., 1964, Manuel d'archéologie égyptienne. Tome IV, Bas-reliefs et peintures. Scènes de la vie quotidienne, Paris.

Vernus, P., 1982, s. v. Mesen, Lexikon der Ägyptologie IV, col. 108-109.

Vernus, P., 1991, «Ménès, Achtoès, I'hippopotame et le crocodile. Lecture structurale de l'historiographie égyptienne », dans U. Verhoeven \& E. Graefe (éd.), Religion und Philosophie im Alten Ägypten. Festgabe für Philippe Derchain zu seinem 65. Geburtstag am 24. Juli 1991, Leuven, p. 331-339. 
WiLD, H., 1948, « Choix d'objets pré-pharaoniques appartenant à des collections de Suisse », Bulletin de l'Institut français d'archéologie orientale 48, p. $1-58$.

WILD, H., 1966, Le tombeau de Ti. Vol. III : la chapelle (deuxième partie), Cairo.

WiLkinson, T.A.H., 1999, Early Dynastic Egypt, London - New York.

Wilkinson, T.A.H., 2000, Royal Annals of Ancient Egypt. The Palermo Stone and its associated fragments, London.

Williams, B. \& Logan, T., 1987, « The Metropolitan Museum Knife Handle and Aspects of Pharaonic Imagery before Narmer », Journal of Near Eastern Studies 46, p. 245-285.

WinkLeR, H.A., 1938, Rock-Drawings of Southern Upper Egypt. Vol. I, London.

YoyotTe, J., 2005, « Hippopotame », dans P. Vernus \& J. Yoyotte, Bestiaire des pharaons, Paris, p. 248-263. 